\title{
Control of a DC motor using algebraic derivative estimation with real time experiments
}

\author{
R. Morales, J.A. Somolinos, H. Sira-Ramírez
}

This paper presents an experimental control scheme for DC motors which combines an overlapping implementation of the algebraic derivative estimation method and a disturbance estimator based on the aforementioned algebraic derivative method. The methodol-ogy only requires the measurement of the angular position of the motor and the voltage input to the motor. The main advantages of the proposed approach are: it is independent of the motor's initial conditions, the methodology is robust to Coulomb friction effects, it does not require any statistical knowledge of the noises that corrupt the data, the deriva-tive estimation process does not require initial conditions or dependence between the sys-tem input and output, and the algorithm is computed online and in real time. The effectiveness of the proposed controller has been verified by means of computer simula-tions and it has also been experimentally implemented on a laboratory prototype with excellent results in both, stabilization and trajectory tracking tasks.

\section{Introduction}

High performance motor drive systems are of primary importance in any industrial application [1]. The DC motor is an essential source of movement in electromechanical systems which are not too powerful [2]. Its main advantage is that it is easy to control the speed or position with a wide adjustable range in order to follow a predetermined time trajectory under different load inputs [3]. Extensive research efforts have been carried out in the past, and various applications can be found in literature: Applications include robot manipulators [4], positioning tables [5], liquid pumps [6], overhead crane mechanisms [7] or disk motion control [8], among many others. The study of effective control methods which exploit the high-speed and high accuracy positioning/tracking performance of DC motors has been the subject of sustained interest for many years. High speed operation is usually required to achieve high productivity, and precision/accuracy becomes more and more rigorous because of the reduced size of the modern electromechanical applications [9]. In [10] Eker combined traditional root mean square errors with discrete time identification algorithms for the on-line control of a mechanical system. Olsson et al. [11] proposed the control of a DC motor using the Coulomb friction estimation and its corresponding compensation. Nouri et al. [12] studied the problem of controlling the speed of a DC motor by using recurrent neural networks, and a sliding mode control with a PID type of sliding surface is implemented in [13]. In recent years, algebraic techniques have been developed for the fast, on line, reliable estimation, or identification, of system parameters, states, failures and input perturbations. The fundamentals of the approach, for the linear system case can be found in the works of Fliess and Sira-Ramírez [14-16], and one of the procedures for parameter identification of a DC Motor model was presented in [17]. 
In this paper, a new control scheme is proposed for the trajectory tracking of a DC motor in the presence of unknown nonlinear effects caused by Coulomb friction, model parametric uncertainties and possible noisy effects in the input control and the output signal. It employs a faster, non-asymptotic, algebraic approach for the determination of time derivative estimates of a signal under additive noise levels and it is endowed with a compensation term based on an algebraic derivative disturbance estimator. The algebraic derivative method relies on a truncated Taylor series approximation of an analytical time signal and its representation as a chain of integrators. The use of operational calculus permits the influence of initial values to be eliminated, and a triangular system of equations is obtained which allows the respective signal time derivatives to be solved up to a certain desired order. The result is a general representation of a linear, time-varying output equation that allows an online-estimation of the required time derivatives [18]. As this approximation is only valid during a certain finite time interval, a periodic resetting of the calculations is necessary. The use of two parallel overlapping estimators to reduce the effects of the computational resettings was presented in [19]. The estimation of the disturbance values and their corresponding compensation in the control system is very important in precision mechanisms because their nonlinear behavior may result in steady state errors, limit cycles, or poor performance [11].

The paper is structured as follows: Section 2 illustrates the dynamic model of the DC servomotor and the problem formulation. Section 3 briefly introduces the theory behind the algebraic derivative method and the notion of an overlapping estimator as a means to improve the quality of the derivative estimators under noisy conditions. Section 4 is devoted to deriving the feedforward controller, based on algebraic derivative and parameter estimation, which is used in the position control of a DC motor. The velocity and the parameter estimation is carried out by means of an overlapping algebraic derivative estimator. This section shows that the proposed controller produces an asymptotically exponentially convergent tracking error behavior towards the origin of coordinates in the error space. In Section 5, comparisons between a PD controller with Coulomb compensation and the robust feedforward controller are illustrated to evaluate the performance and remarkable improvements in the system response. Section 6 describes a laboratory experimental setup and presents the results obtained with the proposed control algorithm. Finally, Section 7 is devoted to the conclusions of this article and proposals for future work.

\section{The DC motor model and problem formulation}

\subsection{DC motor dynamics}

This section is devoted to the background results of the linear model of the DC motor. It is assumed that the linear model is affected by an unknown perturbation input caused by Coulomb friction effects (see [11]). The DC motor is assumed to be fed via a servo-amplifier with a current, inner loop, control. The main dynamic equation of the system is obtained from Newton's Second Law:

$k u=J \ddot{\bar{\theta}}_{m}+v \dot{\bar{\theta}}_{m}+\bar{\Gamma}_{c}\left(\dot{\bar{\theta}}_{m}\right)$,

where $u$ is the motor input voltage that acts as the control variable for the system. This is the input to a servo-amplifier which controls the input current to the motor by means of an internal PI current controller (see Fig. 1(a)). The electrical dynamics can be ignored because it is much faster than the mechanical dynamics of the motor, signifying that the servo-amplifier can be considered as a constant relation, $k_{e}$, between the voltage and the current to the motor: $i_{m}=k_{e} u$ (see Fig. 1(b)), where $i_{m}$ is the armature circuit current, $k_{e}$ includes the gain of the amplifier, $\tilde{k}$, and $R$ is the input resistance of the amplifier circuit. The magnitude $J$ is the inertia of the motor and gear $\left[\mathrm{kg} \mathrm{m}^{2}\right], v$ is the viscous friction coefficient and $\bar{\Gamma}_{c}$ is the unknown Coulomb friction torque which affects the motor dynamics. This nonlinear friction term is considered as a perturbation and obeys the following equation:

$\bar{\Gamma}_{c}=\bar{\Gamma}_{\text {Coul }} \cdot \operatorname{sign}\left(\dot{\bar{\theta}}_{m}\right)$,

where $\bar{\Gamma}_{\text {Coul }}$ is the static friction value which the motor torque must exceed to start the axis motions. The parameter $k$ is the electromechanical constant of the motor servoamplifier system and $\ddot{\bar{\theta}}_{m}$ and $\dot{\bar{\theta}}_{m}$ are the angular acceleration of the motor and the angular velocity of the motor, respectively. The constant factor $n$ is the reduction ratio of the motor gear; thus $\theta_{m}=\bar{\theta}_{m} / n$, where $\theta_{m}$ is the position of the motor gear and $\bar{\theta}_{m}$ is the position of the motor shaft. Moreover, $\Gamma_{c}=\bar{\Gamma}_{c} n$, where $\Gamma_{c}$ is the Coulomb friction torque in the motor gear.

The total torque delivered to the motor $\Gamma_{T}$ is directly proportional to the armature circuit in the form $\Gamma_{T}=k_{m} i_{m}$, where $k_{m}$ is the electromechanical constant of the motor. The electromechanical constant of the motor servo-amplifier system is therefore $k=k_{m} k_{e}$. Manipulating expression (1) one obtains:

$u=\frac{J}{k} \ddot{\bar{\theta}}_{m}+\frac{v}{k} \dot{\bar{\theta}}_{m}+\frac{\bar{\Gamma}_{c}}{k}$.

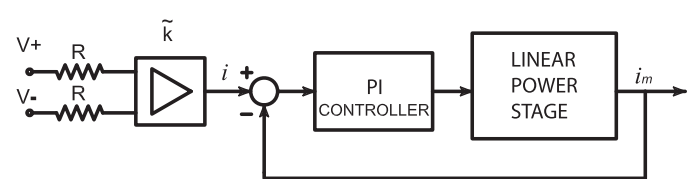

(a)

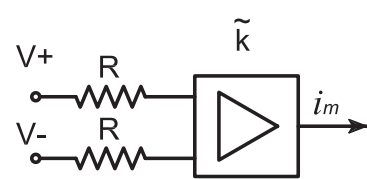

(b)

Fig. 1. (a) Complete amplifier scheme; (b) equivalent amplifier scheme. 
In order to simplify the developments, let $K=k / n, A=K / J$, $B=v / J$. If substitutions are made in the previous expression, the following DC motor dynamics are obtained in terms of variables on the side of the motor gear:

$A u=\ddot{\theta}_{m}+B \dot{\theta}_{m}+\frac{\Gamma_{c}}{J n^{2}}$.

\subsection{Problem formulation}

Consider the previously described DC motor dynamics presented in (4). Given a desired sufficiently smooth reference trajectory $\theta_{m}^{*}(t)$ for the position of the motor gear, $\theta_{m}$, and taking into account the possibly noisy measurements of the input signal, $u$, and of the output signal, $\theta_{m}$, and possible unknown nonlinear effects caused by Coulomb friction and model parametric uncertainties, devise an output feedback controller, $u$, which guarantees the asymptotic tracking of $\theta_{m}^{*}(t)$ by the system output $\theta_{m}$.

\section{Algebraic time derivative calculations}

In an observable system, the state estimation problem is intimately related to the problem of computing the successive time derivatives of the output and input signals in a sufficiently large number (see [20]). A non-asymptotic algebraic procedure for the approximate estimation of the system states is briefly presented below. The method is based on results from differential algebra and furnishes some general formulae for the time derivatives of a measurable signal. Some other interesting contributions also propose non-asymptotic approaches to state estimation in dynamical systems (See, for example, the works [21-23]).

\subsection{Mathematical framework}

Some steps of the derivation presented in [18] are now recalled for the sake of simplicity. Let us consider an arbitrary, analytic time signal $y(t), y: \mathbb{R}_{0}^{+} \rightarrow \mathbb{R}$. In the vicinity of $t=t_{r}$, the following $(N-1)$ th order polynomial model for the approximation of the signal $y(t)$ is valid

$y(t) \approx \tilde{y}(t)=\sum_{i=1}^{N} \frac{y^{(i-1)}\left(t_{r}\right)}{(i-1) !}\left(t-t_{r}\right)^{i-1} 1\left(t-t_{r}\right)$.

It is possible to differentiate $\tilde{y}(t)$ at least $N$-times with respect to time, so as to obtain an expression that is identical to zero. In the operator domain $(\widetilde{Y}(s)=\mathcal{L}[\tilde{y}(t)])$, this reads as:

$$
\left.s^{N} \widetilde{Y}(s)-\sum_{i=1}^{N} s^{N-i} y^{(i-1)}\left(t_{r}\right)\right) \cdot e^{-s t_{r}}=0,
$$

which involves only standard rules of operational calculus, and the expression $e^{-s t_{r}}$ originates from the time shift to $t=t_{r}$. Taking into account that $e^{-s t_{r}} \neq 0$, the following result is obtained:

$s^{N} \widetilde{Y}(s)-\sum_{i=1}^{N} s^{N-i} y^{(i-1)}\left(t_{r}\right)=0$.
In order to eliminate the initial conditions, $y^{(i-1)}\left(t_{r}\right)$, it is necessary to differentiate $N$ times with respect to the complex operator $s$. One obtains:

$\frac{d^{N}}{d s^{N}}\left(s^{N} \widetilde{Y}(s)\right)=0$

Since the highest degree of $s$ in the above expression is $N$, it is easy to see that the $N$ th time derivative of $\tilde{y}(t)$ can be expressed in terms of lower order derivatives. Pre-multiplication by an iterated integration operator therefore results in a recursive system with which to determine all required time derivatives of $\tilde{y}(t)$, i.e., the expressions given by

$s^{-v} \frac{d^{N}}{d s^{N}}\left(s^{N} \widetilde{Y}(s)\right)=0, \quad v=N-1, N-2, \ldots, N-\eta$

contains, respectively, implicit information on the first, second, ..., $(N-1)$ th derivatives of $y(t)$ in an approximate manner. According to [18] the following result is obtained,

$$
\begin{aligned}
\tilde{y}^{(i)}(t)= & \frac{1}{\left(t-t_{r}\right)^{i}} \frac{(N+i-1) !}{(N-i-1) ! i !} y(t) \\
& +\sum_{j=1}\left(\begin{array}{l}
N+i-j-1 \\
i-j
\end{array}\right) \frac{(N-j-1) !}{(N-i-1) !} \frac{z_{j}(N, t)}{\left(t-t_{r}\right)^{N+i-j}}, \\
i= & 1, \ldots, v
\end{aligned}
$$

where the filter states $z_{j}(N, t), j=1, \ldots, N-1$, obey

$$
\begin{aligned}
& \dot{z}_{j}(N, t)=\left(\begin{array}{l}
N \\
j+1
\end{array}\right)^{2}(j+1) !(-1)^{j}\left(t-t_{r}\right)^{N-j-1} y(t) \\
& +z_{j+1}(N, t), \quad j=1, \ldots, N-2, \\
& \dot{z}_{N-1}(N, t)=N !(-1)^{N-1} y(t),
\end{aligned}
$$

which is an $(N-1)$ th order time-varying, linear filter with homogeneous initial conditions $z_{j}\left(N, t_{r}\right)=0$ for $j=1, \ldots$, $N-1$. Note that at time $t=t_{r}$ the above formulae yields an indetermination. In fact, the finite precision of the numerical processors signifies that the computation will not be appropriately defined in a small interval of time of the form: $\left[t_{r}, t_{r}+\epsilon\right)$. The formulae for $\dot{\tilde{y}}, \ddot{\tilde{y}}$, etc., are therefore valid for $t \geqslant t_{r}+\epsilon>0$. During the interval of time $\left[t_{r}, t_{r}+\epsilon\right)$, we may replace their values with arbitrary constant values or with appropriate function approximations (see [18] for details). The issue of how and when to update, or re-initialize, the computations is examinated next.

\subsection{Calculations resettings}

The validity of the formulae for the estimates of $y^{(i)}(t)$ in the open time interval $\left[t_{r}+\epsilon, t\right)$ becomes questionable as $t$ grows, owing to the approximate nature of the truncated Taylor series expansion adopted. The calculations need to be reset at a particular finite time $t_{r}$. In this work, the reset policy of equidistant intervals is used. This strategy consists of choosing time intervals whose length $T$ may be arbitrarily fixed at the outset. The validity of the formulae in each interval of the form $\left[t_{r}+\epsilon, t_{r}+T\right)$ is thus assumed. Clearly, $T \gg \epsilon$. Naturally, this entitles choosing a small value for $T$. The determination of $T$ may require some additional off-line trial and error runs. This strategy is obviously highly dependent on the encoding system and 
requires judgment rather than an objective criterion evaluation. Alternatively, dynamic reset policies can be chosen to achieve the automatic resetting of the calculations when the actual values of such derivatives are not known beforehand (see [18] for details).

\subsection{Noise with unknown statistical properties}

It is assumed that $y(t)$ is perturbed by an additive zero mean noise with otherwise unknown statistical properties. In order to enhance the signal-to-noise ration (SNR), we simultaneously filter the numerator $n(t)$ and denominator $d(t)$ using the same low pass filter [24]. If advantage is taken of the estimator rational form in (10), then the quotient will not be affected by the filters. This invariance is emphasized with the use of the different notations in the frequency and time domain such as:

$y^{(i)}(t)=\frac{n_{f}(t)}{d_{f}(t)}=\frac{F(s) n(t)}{F(s) d(t)}$,

where $n_{f}(t)$ and $d_{f}(t)$ are the filtered numerator and denominator, and $F(s)$ is the being filter used. The choice of this filter depends on the a priori knowledge of the system that is available. Nevertheless, if such knowledge does not exist, pure iterated integrations of the form $1 / s^{k}, k \geqslant 1$ may be utilized whenever zero mean high frequency noise is assumed. This hypothesis has been motivated by recent developments in the non-standard analysis of a new non-stochastic noise theory (see [25] for further details).

\subsection{Overlapping derivative estimators technique}

From Eq. (10) it is clear that, for numerical reasons, a small interval of time $\epsilon$ has to elapse before the results of the estimators become accurate (note the singularity at $\left.t=t_{r}\right)$. Moreover, depending on the amount of noise $\xi(t)$ associated with the measured signal $y(t)$, a certain period of integration time is also needed for the filters to attenuate the noise effects. Furthermore, the Taylor polynomial approximation signifies that the estimation of the signal, $\tilde{y}(t)$, will start to diverge from the true signal $y(t)$ when the time difference $t-t_{r}$ becomes significantly large. For a proper update of the derivative estimation, a reset of the filter states to zero may be necessary at some instant, implying a new $\epsilon$-period of integration time before we again achieve accurate estimation results. In order to enlarge the intervals of validity of the estimation scheme proposed, the following estimation policy is introduced: two estimators run simultaneously but in an overlapping fashion so as to obtain valid results at all times except in the very first $\epsilon$-interval. The re-initialization of each identifier is separated by a time interval of duration $T / 2$. This can be performed by defining two time lines, $t_{1}$ and $t_{2}$, which are defined as follows:

$t_{1}=t \bmod T$,

$t_{2}=t-T / 2 \bmod T$,

where the time line for the first identifier is defined as $t_{1}$ and, similarly, $t_{2}$ for the second identifier. The first identifier is re-initialized when $t_{1}=0$ and the second identifier when $t_{2}=0$. This policy is called $a$ switched overlapping estimators technique. Fig. 2 depicts the time lines for both identifiers. An alternative approach to algebraic derivative estimation based on a receding horizon strategy is presented in [26] and a experimental application in a brake-testbench is presented in [27]. In this method, the estimation values of the parameters are exactly the same than the proposed in the overlapping derivative estimation technique. The approach exhibits efficiency in the calculation of the parameters to be estimated because it avoids the numerical saturation of the numerator and the denominator of each parameter to be estimated. On the other hand, the proposed overlapping derivative estimation technique resets one of the estimators while the original remains active and vice versa (i.e., it resets the previously active one while the second estimator is still valid and non-saturated). This allows the avoidance of discontinuities in the derivative calculation process. To obtain an optimum performance of the algebraic derivative estimation, the choice of a specific time function allows one to take influence on the sensitivity to noise and the adaptation speed (see [28] for details). In the work presented in this manuscript, we opt of the use of the switched overlapping estimators technique because its simplicity and the results obtained are good enough for our purposes.

\section{Controller design}

This section presents the proposed feedback control approach for the solution of the trajectory tracking control of DC motors. The proposed method has the following advantages: (a) It does not require any statistical knowledge of the noises corrupting the data; (b) the derivative estimation does not require initial conditions or dependence between the system input and output and (c) the algorithm is computed on-line and in real time. A robust feedforward proportional derivative (PD) controller has been implemented which utilizes an overlapping algebraic derivative estimator and an algebraic derivative disturbance estimator. This sort of scheme is used because the derivative action reduces the overshooting that produces the integral action that appears in Proportional Integral (PI) Controllers and which could destabilize the system

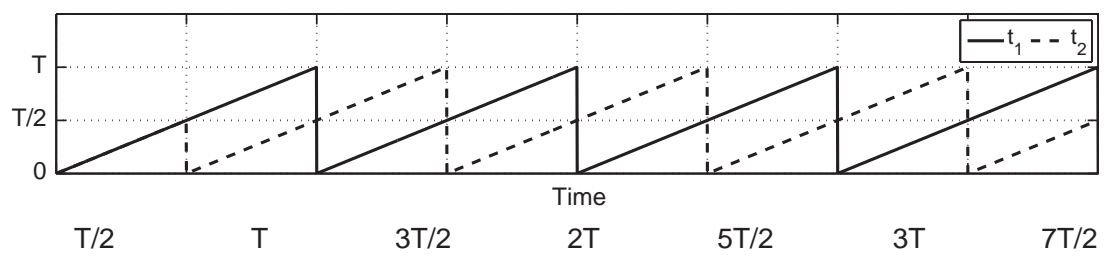

Fig. 2. Time setting for each identifier. 


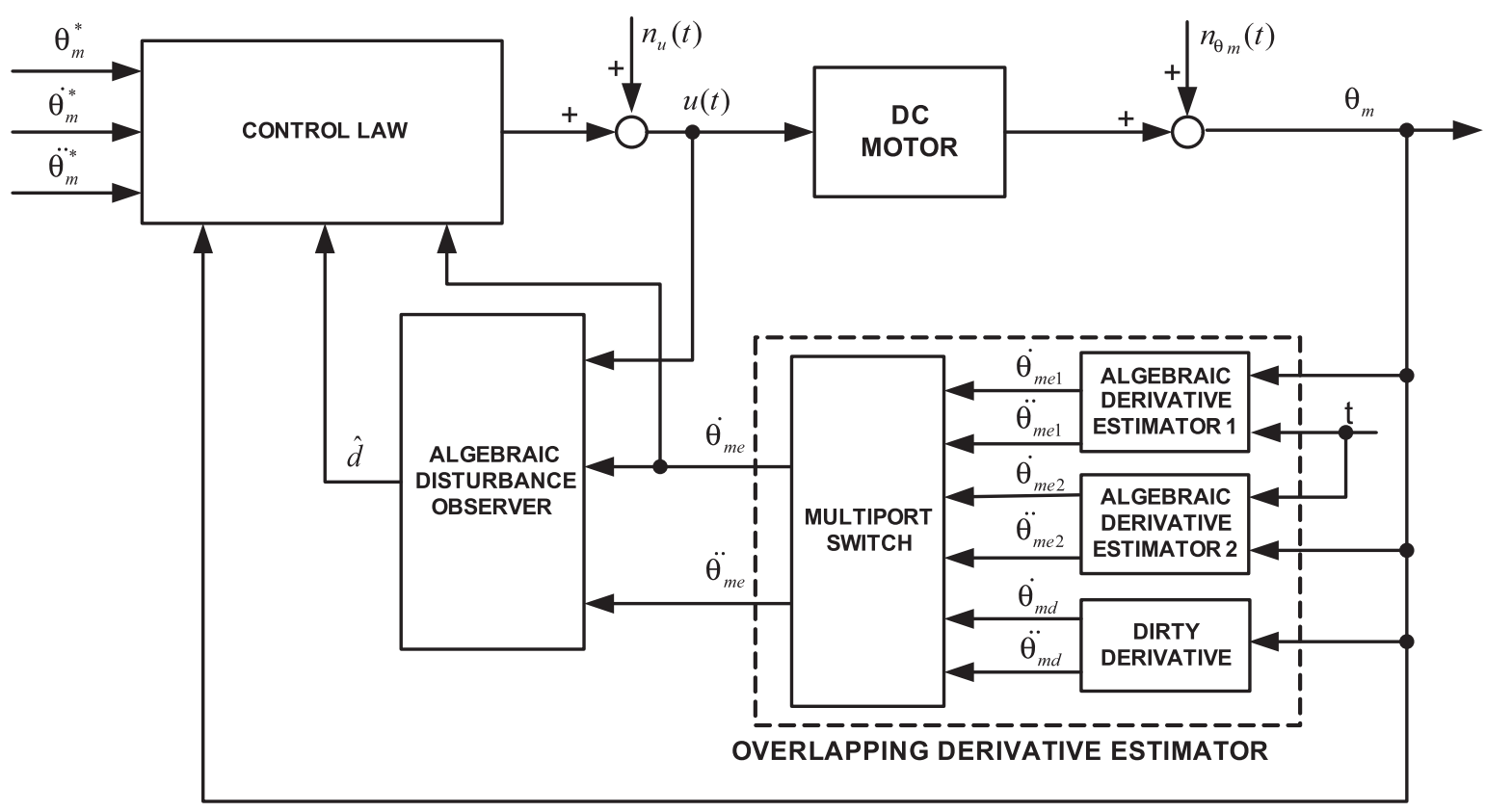

Fig. 3. Designed control scheme.

when high integral gain values are used. In comparison, the classical PD controller eliminates such overshooting but includes a steady state error when constant perturbations affect the system dynamics. The controller proposed in this work, which is illustrated in Fig. 3, achieves excellent results for both, stabilization and trajectory tracking tasks. The different parts of the proposed control scheme are explained in the following subsections.

\subsection{Feedback controller}

If the dynamical model of the motor presented in (4) is considered, then the voltage applied to the motor can be written as

$u=\frac{1}{A}\left(\ddot{\theta}_{m}+B \dot{\theta}_{m}\right)+d$,

where $d$ is a perturbation that includes the effects produced by the Coulomb friction, $\Gamma_{c} /\left(J n^{2} A\right)$ which depends only on the sign of the angular velocity, and possible model parametric uncertainties. The controller to be designed must be robust with regard to these unknown piecewise disturbances that affect the motor dynamics. A direct exact linearization based feedback controller design with which to accomplish the tracking of a given desired output motor gearbox angular position, $\theta_{m}^{*}$, is given by:

$u(t)=\frac{1}{A}\left[\ddot{\theta}_{m}^{*}+B \dot{\theta}_{m}^{*}-k_{1}\left(\dot{\theta}_{m}-\dot{\theta}_{m}^{*}\right)-k_{0}\left(\theta_{m}-\theta_{m}^{*}\right)\right]$ $+d$

where the control law can be uniquely specified if the parameter $d$ is known. In this case, the closed loop tracking error, $e_{\theta_{m}}=\theta_{m}-\theta_{m}^{*}$, evolves governed by

$\ddot{e}_{\theta_{m}}+\left(B+k_{1}\right) \dot{e}_{\theta_{m}}+k_{0} e_{\theta_{m}}=0$
The parameters $\left\{k_{1}, k_{0}\right\}$ can be designed by choosing to place the closed loop poles in a desired location in the left half of the complex plane. The two poles can be located at the same point of the real line, $s=-p$, where $p$ is a strictly positive constant parameter. The following characteristic equation is achieved:

$(s+p)^{2}=s^{2}+2 p s+p^{2}=0$.

The characteristic equation of the closed loop system is,

$s^{2}+\left(B+k_{1}\right) s+k_{0}=0$.

If terms of the same degree are equated in (17) and (18) then the value of the coefficients $\left\{k_{1}, k_{0}\right\}$ is obtained. These are given by:

$k_{1}=2 p-B ; \quad k_{0}=p^{2}$.

We have thus proven the following proposition:

Proposition 1. Given the second order perturbed system of the DC motor presented in (4), where it is assumed that the velocity of the motor gear, $\dot{\theta}_{m}$, is perfectly measurable, and the magnitude $d$ is known, and given a sufficiently smooth reference trajectory $\theta_{m}^{*}(t)$ for the position of the motor gear, $\theta_{m}$, then under the appropriate choice of the design parameters $\left\{k_{1}, k_{0}\right\}$, such that the characteristic polynomial of the closed loop linear system, $p(s)=s^{2}+\left(B+k_{1}\right) s+k_{0}$, is a Hurwitz polynomial, and the controller (15) provides the desired tracking error equilibrium point, $e_{\theta_{m}}(t)=\theta_{m}-\theta_{m}^{*}=0$, with an exponentially asymptotically stable nature.

\subsection{Algebraic derivative estimator}

Only the measured motor position $\theta_{m}$ is used for the synthesis of the proposed feedback-control law (15). However, the controller requires the first time derivative of the 
position of the motor gear, $\dot{\theta}_{m}$, to implement the control law described above, the first and second time derivatives of the position of the motor gear, $\dot{\theta}_{m}$ and $\ddot{\theta}_{m}$ respectively, to compute the algebraic disturbance observer described in Section 4.3, and these signals are not available for measurement. In this section, an estimate of these time derivative signals is carried out via algebraic estimation techniques, as explained in Section 3. The time derivatives of the measured output, $\theta_{m}(t)$ are generated using a truncated Taylor series expansion, up to a sixth order, of $\theta_{m}(t)$ around the re-initialization time $t_{r}$ :

$\tilde{\theta}_{m}(t)=\sum_{i=1}^{5} \frac{\theta_{m}^{(i-1)}\left(t_{r}\right)}{(i-1) !}\left(t-t_{r}\right)^{i-1}$,

which, in the frequency domain, leads to the identity

$\frac{d^{5}}{d s^{5}}\left[s^{5} \Theta_{m}(s)\right]=0$.

Based on the previous developments, the derivative calculations in terms of a time varying linear filter are written (according to expression (11)) as follows:

$\dot{\theta}_{m e}(t)= \begin{cases}\text { arbitraryconstant } & \text { fort } \in\left[\mathrm{t}_{\mathrm{r}}, \mathrm{t}_{\mathrm{r}}+\epsilon\right) \\ \frac{1}{\left(\mathrm{t}-\mathrm{t}_{\mathrm{r}}\right)^{5}}\left[20\left(\mathrm{t}-\mathrm{t}_{\mathrm{r}}\right)^{4} \theta_{\mathrm{m}}(\mathrm{t})+\mathrm{z}_{1}(\mathrm{t})\right] & \text { fort } \geqslant \mathrm{t}_{\mathrm{r}}+\epsilon,\end{cases}$

$\ddot{\theta}_{m e}(t)= \begin{cases}\text { arbitraryconstant } & \text { fort } \in\left[\mathrm{t}_{\mathrm{r}}, \mathrm{t}_{\mathrm{r}}+\epsilon\right) \\ \frac{1}{\left(\mathrm{t}-\mathrm{t}_{\mathrm{r}}\right)^{6}}\left[180\left(\mathrm{t}-\mathrm{t}_{\mathrm{r}}\right)^{4} \theta_{\mathrm{m}}(\mathrm{t})+15 \mathrm{z}_{1}(\mathrm{t})+\left(\mathrm{t}-\mathrm{t}_{\mathrm{r}}\right) \mathrm{z}_{2}(\mathrm{t})\right] & \text { fort } \geqslant \mathrm{t}_{\mathrm{r}}+\epsilon,\end{cases}$

The above formulaes for the estimation of the velocity and the acceleration of the motor gear, $\dot{\theta}_{m}$ and $\ddot{\theta}_{m}$, are valid after a small time interval of duration $\epsilon$ has elapsed from the instant $t=t_{r}$, i.e., during the interval $\left[t_{r}+\epsilon, t\right)$. A new resetting must be carried out when the validity of the approximation becomes questionable. As was explained in Section 3, assuming the fact that $\epsilon \ll t_{r}$ the use of the overlapping derivative estimation technique makes it possible to re-initialize without singularities and substantially improves the accuracy of the estimation of the derivatives of the signal. This is carried out by using two identifiers, in a resetting mode configuration, such that the re-initialization of each identifier is separated in a time interval of $T / 2 \mathrm{~s}$ (see expression (13)). Fig. 2 shows the settings of each of the two time lines. It is necessary to denote the first and second estimations for the velocity of the motor gear as given by:

where exposition, $\dot{\theta}_{m 1 e}$ and $\dot{\theta}_{m 2 e}$ and the first and second estimations of the acceleration of the motor gear as $\ddot{\theta}_{m 1 e}$ and $\ddot{\theta}_{m 2 e}$. Furthermore, during the first $\epsilon$-interval in which the results of the algebraic derivative estimators are not yet valid, filtered dirty first and second time derivatives of $\theta_{m}$ are used instead of an arbitrary constant. The reason for this is that these terms are in charge of capturing the behavior of the system in the period of time $[0, \epsilon]$ in the simulation/experiment, which could be critical in systems with initial high input overshooting or in the control of systems of an unstable nature. The estimations of the velocity and acceleration of the motor gear based on the two identifiers scheme, the filtered dirty time derivatives, $\dot{\theta}_{m_{e}}$ and $\ddot{\theta}_{m_{e}}$, are therefore

$\dot{\theta}_{m e}(t)= \begin{cases}\dot{\theta}_{m_{\text {dirty }}}(t) & \text { for } 0 \leqslant t \leqslant \epsilon, \\ \dot{\theta}_{m 2_{e}}(t) & \text { for }(0 \leqslant t \bmod T<T / 2) \operatorname{AND}(t>\epsilon), \\ \dot{\theta}_{m 1_{e}}(t) & \text { for }(T / 2 \leqslant t \bmod T<T) \operatorname{AND}(t>\epsilon),\end{cases}$
$\ddot{\theta}_{m e}(t)= \begin{cases}\ddot{\theta}_{m_{\text {dirty }}}(t) & \text { for } 0 \leqslant t \leqslant \epsilon, \\ \ddot{\theta}_{m 2_{e}}(t) & \text { for }(0 \leqslant t \bmod T<T / 2) \operatorname{AND}(t>\epsilon), \\ \ddot{\theta}_{m 1_{e}}(t) & \text { for }(T / 2 \leqslant t \bmod T<T) \operatorname{AND}(t>\epsilon) .\end{cases}$

\subsection{Algebraic disturbance observer}

In the time domain, the disturbance effects that act in the system can be expressed by means of the following differential equation:

$d=u(t)-\frac{1}{A}\left(\ddot{\theta}_{m}-B \dot{\theta}_{m}\right)$

The computation of the time derivatives of $\theta_{m}$ and the voltage input control, $u(t)$, clearly makes it possible to reconstruct the disturbance parameter $d$. Indeed, an estimate of the signal $d$ would be obtained using

$\hat{d}=u(t)-\frac{1}{A}\left(\ddot{\theta}_{m e}-B \dot{\theta}_{m e}\right)$,

where $\dot{\theta}_{m e}$ and $\ddot{\theta}_{m e}$ are the first and second time derivatives estimates of the measured output signal $\theta_{m}$.

Finally, we conclude this section by stating our main result, which has been proven throughout the previous 


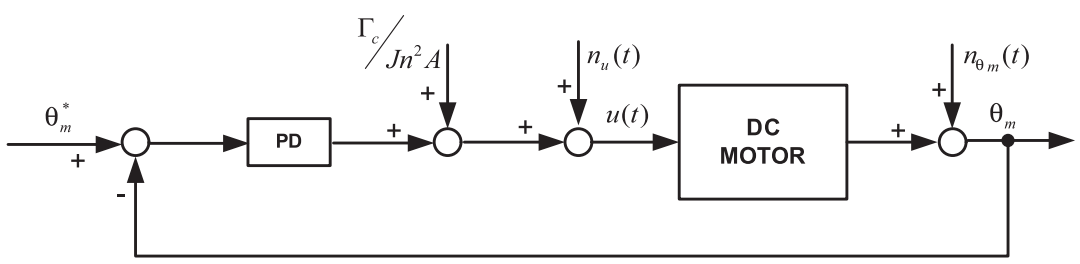

Fig. 4. PD control scheme with Coulomb compensation.

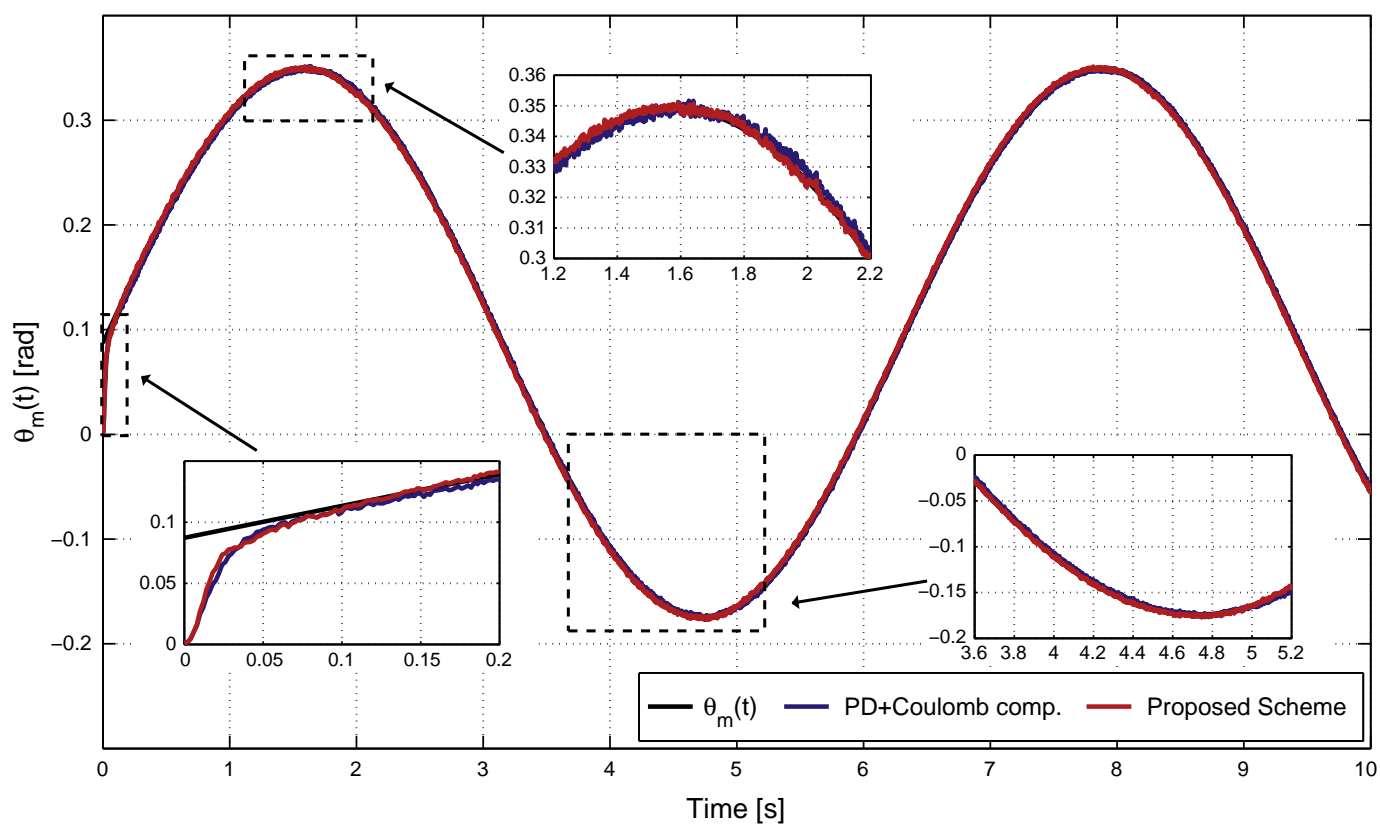

Fig. 5. Sinusoidal trajectory - evolution of the DC motor position motor gear, $\theta_{m}$, using a PD controller with Coulomb compensation and the robust feedforward PD controller when large initial errors appear and when the Coulomb friction effects are not precisely known.

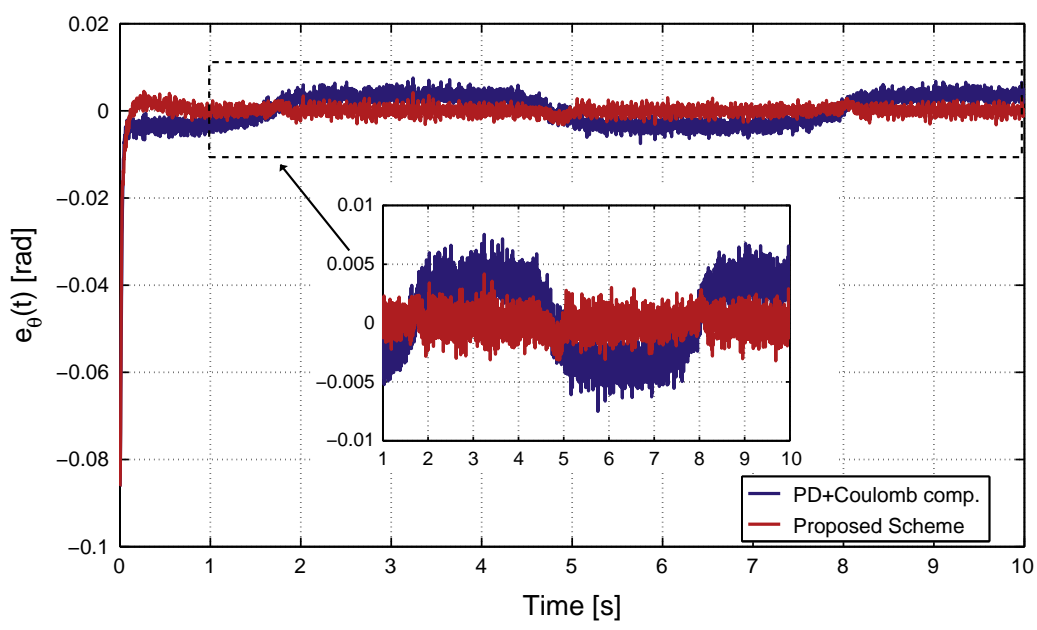

Fig. 6. Sinusoidal trajectory - evolution of the tracking error, $e_{\theta_{m}}=\theta-\theta_{m}$, using a PD controller with Coulomb compensation and the robust feedforward $\mathrm{PD}$ controller in the face of large initial errors appear, unprecise knowledge of the Coulomb's friction effects, noisy measurements and controller gain mismatches. 

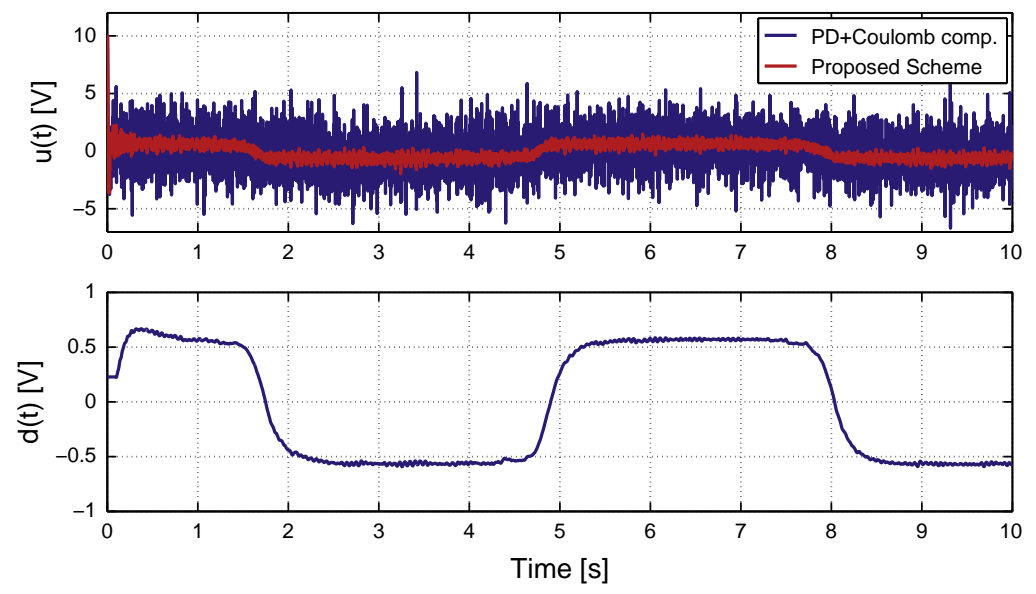

Fig. 7. Sinusoidal trajectory - estimation of the parameter, $d(t)$, and the input control, $u(t)$, using a PD controller with Coulomb compensation and the robust feedforward PD controller confronted with the appearance of large initial errors, imprecise knowledge of the Coulomb friction effects, noisy measurements and controller gain mismatches.
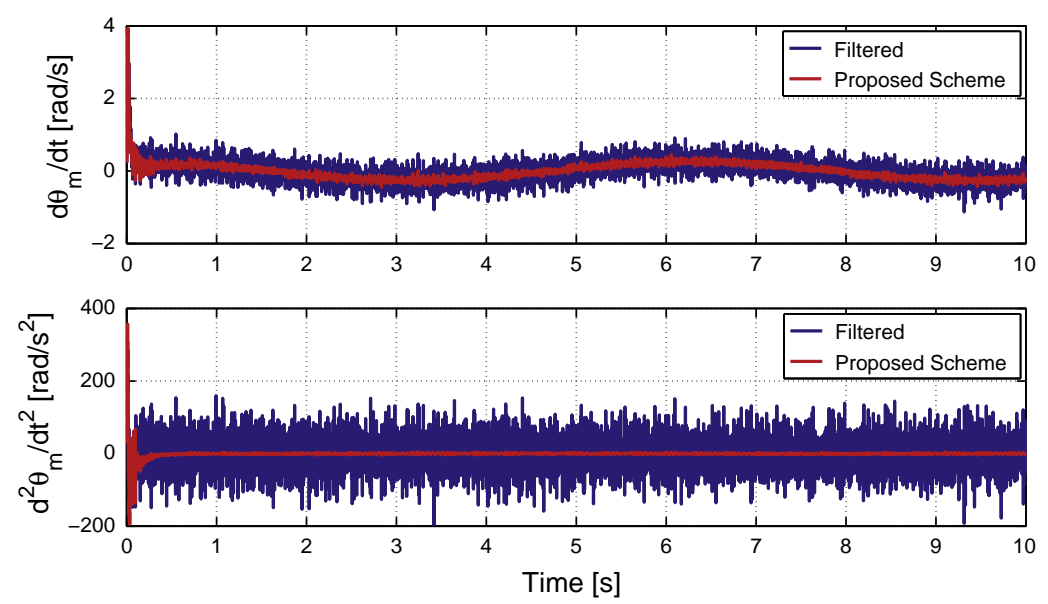

Fig. 8. Sinusoidal trajectory - estimation of the DC motor gear velocity, $\dot{\theta}_{m}$ and estimation of the DC motor gear acceleration, $\ddot{\theta}_{m}$ when confronted with the appearance of large initial errors, imprecise knowledge of the Coulomb friction effects, noisy measurements and controller gain mismatches.

Table 1

Sinusoidal trajectory - performances of control methods.

\begin{tabular}{llll}
\hline Control method & ISE & IAE & ITAE \\
\hline PD + Coulomb compensation & $0.21 \times 10^{-3}$ & $31.40 \times 10^{-3}$ & $15 \times 10^{-2}$ \\
Robust feedforward PD & $0.10 \times 10^{-3}$ & $9.46 \times 10^{-3}$ & $3.5 \times 10^{-2}$ \\
\hline
\end{tabular}

Proposition 2. Given a sufficiently smooth reference trajectory, $\theta_{m}^{*}(t)$, for the position of the motor gear, $\theta_{m}(t)$, in the $D C$ motor system dynamics:

$u=\frac{1}{A}\left(\ddot{\theta}_{m}+B \dot{\theta}_{m}\right)+d$,

and assuming that only the measurement of the position of the motor gear, $\theta_{m}(t)$ is available, and that the applied control input, $u(t)$, is perfectly known, then, the feedback controller:

$$
\begin{aligned}
& u(t)=\frac{1}{A}\left[\ddot{\theta}_{m}^{*}+B \dot{\theta}_{m}^{*}-k_{1}\left(\dot{\theta}_{m e}-\dot{\theta}_{m}^{*}\right)-k_{0}\left(\theta_{m}-\theta_{m}^{*}\right)\right]+\hat{d}, \\
& \hat{d}=u(t)-\frac{1}{A}\left(\ddot{\theta}_{m e}-B \dot{\theta}_{m e}\right), \\
& \dot{\theta}_{m e}(t)= \begin{cases}\dot{\theta}_{m_{\text {dirty }}(t)} \text { for } 0 \leqslant t \leqslant \epsilon, \\
\dot{\theta}_{m_{e}}(t) \text { for }(0 \leqslant t \bmod T<T / 2) \text { AND }(t>\epsilon), \\
\dot{\theta}_{m l_{e}}(t) \text { for }(T / 2 \leqslant t \bmod T<T) \text { AND }(t>\epsilon),\end{cases} \\
& \ddot{\theta}_{m e}(t)= \begin{cases}\ddot{\theta}_{m_{\text {dirty }}}(t) & \text { for } 0 \leqslant t \leqslant \epsilon, \\
\ddot{\theta}_{m_{e}}(t) & \text { for }(0 \leqslant t \bmod T<T / 2) \operatorname{AND}(t>\epsilon), \\
\ddot{\theta}_{m l_{e}}(t) & \text { for }(T / 2 \leqslant t \bmod T<T) \operatorname{AND}(t>\epsilon),\end{cases}
\end{aligned}
$$




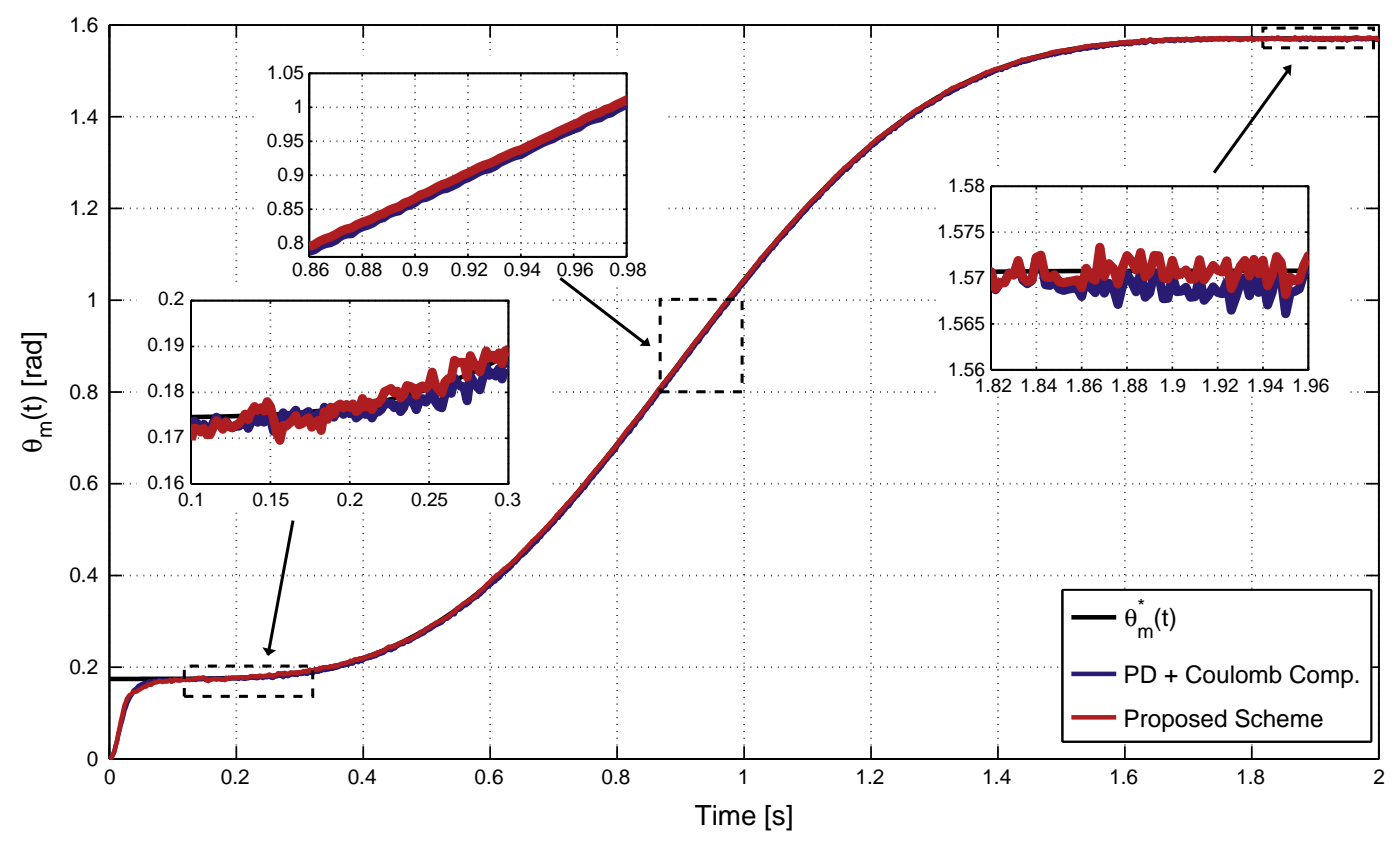

Fig. 9. Bezier trajectory - evolution of the DC motor position motor gear, $\theta_{m}$, using a PD controller with Coulomb compensation and the robust feedforward PD controller when large initial errors appear and when the Coulomb friction effects are not precisely known.

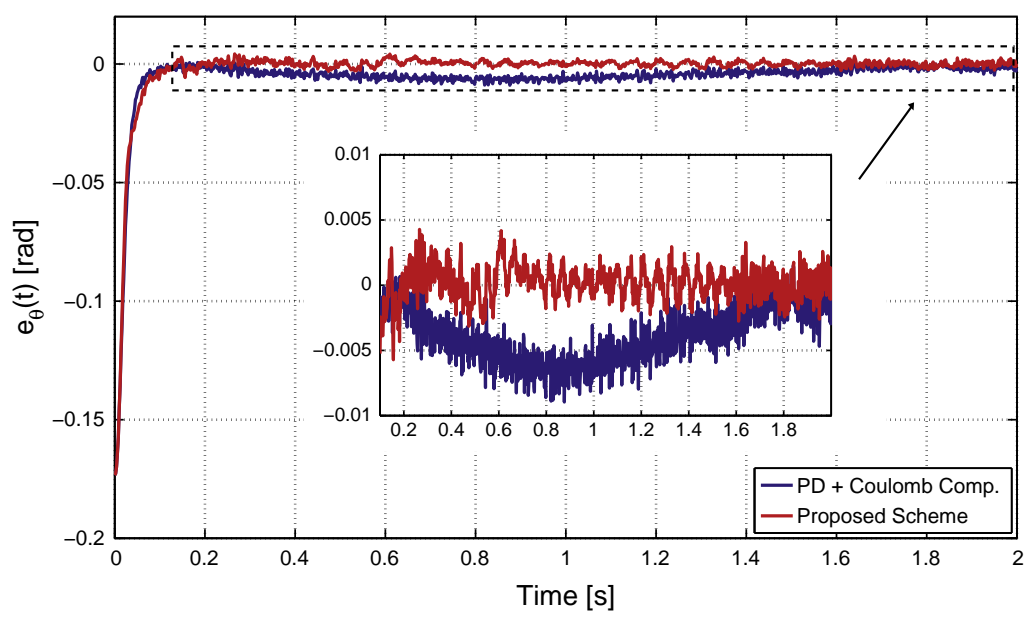

Fig. 10. Bezier trajectory - evolution of the tracking error, $e_{\theta_{m}}=\theta-\theta_{m}$, using a PD controller with Coulomb compensation and the robust feedforward PD controller when confronted with the appearance of large initial errors, imprecise knowledge of the Coulomb friction effects, noisy measurements and controller gain mismatches.

produces a closed loop behavior of the tracking error, $e_{\theta_{m}}=\theta_{m}-\theta_{m}^{*}(t)$, which is governed by the linear dynamics

$\ddot{e}_{\theta_{m}}+k_{1} \dot{e}_{\theta_{m}}+k_{0} e_{\theta_{m}}=0$,

whose design coefficients $\left\{k_{1}, k_{0}\right\}$ have been chosen so as to render the origin of the tracking error space at an exponentially stable equilibrium point.

\section{Simulation results}

Numerical simulations were carried out in order to verify the performance of the proposed control law in terms of the quick convergence of the tracking errors to a small neighborhood of zero, smooth transient responses and low control effort when the system is affected by large initial errors, inexact knowledge of the effects produced by the Coulomb friction, noisy measurements and controller gain mismatches. A robustness comparison between the scheme proposed and a PD controller (see Fig. 4) with which to compensate for the Coulomb effects, which is usually considered to be a robust controller in robotics applications owing its simplicity and good performance (see for example [29-33]), is additionally carried out in order to show the improvements made to the controller designed. The transfer function considered for the PD controller was set at $P D(s)=k_{p}+k_{d} s$ whose 

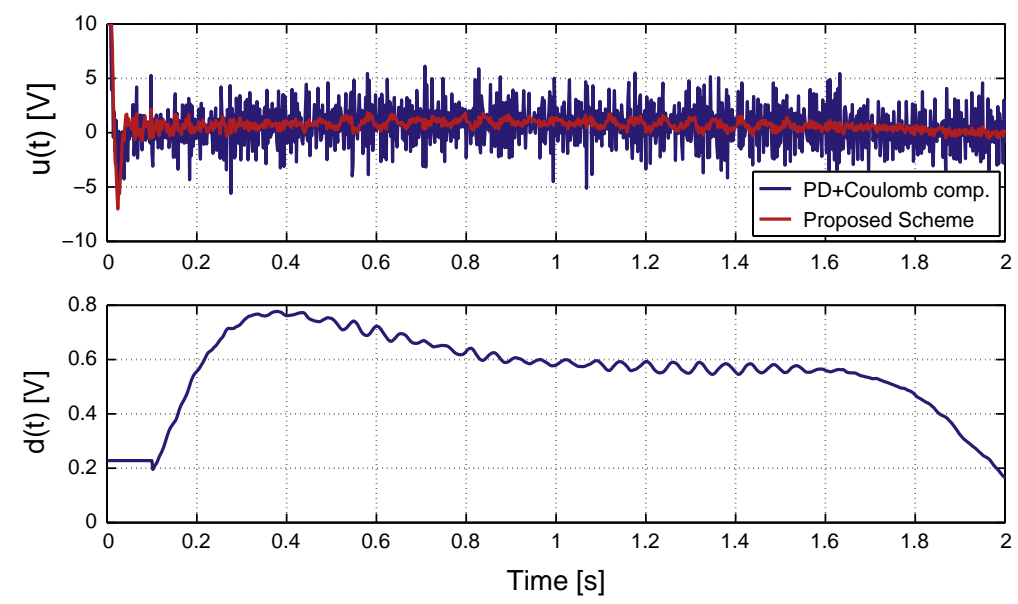

Fig. 11. Bezier trajectory - estimation of the parameter, $d(t)$, and the input control, $u(t)$, using a PD controller with Coulomb compensation and the robust feedforward PD controller when confronted with the appearance of large initial errors, imprecise knowledge of the Coulomb friction effects, noisy measurements and controller gain mismatches.
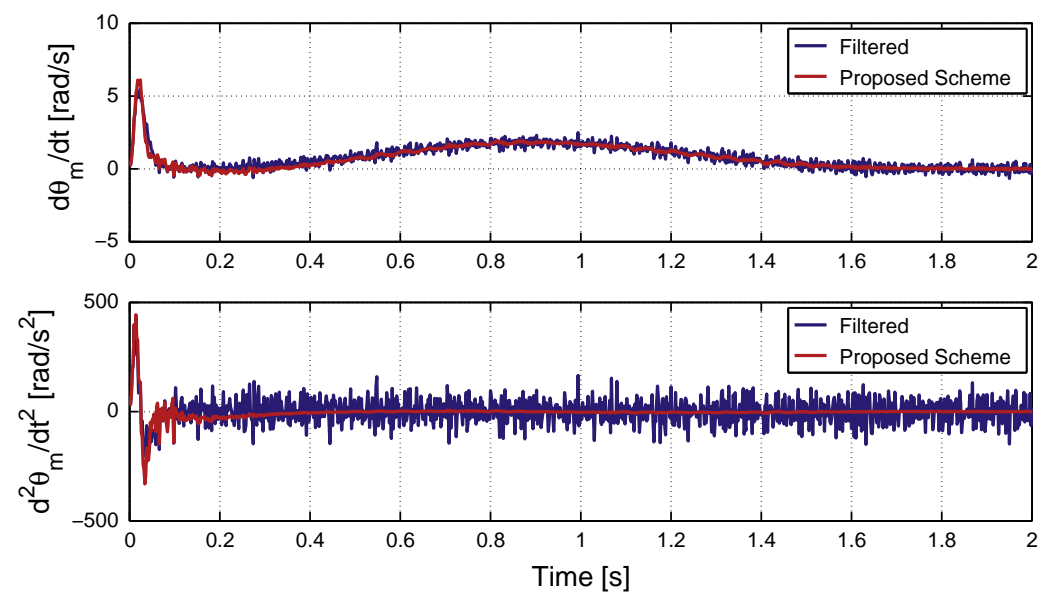

Fig. 12. Bezier trajectory - estimation of the DC motor gear velocity, $\dot{\theta}_{m}$ and estimation of the DC motor gear acceleration, $\ddot{\theta}_{m}$ when confronted with the appearance of large initial errors, imprecise knowledge of the Coulomb friction effects, noisy measurements and controller gain mismatches.

gains $\left\{k_{p}, k_{v}\right\}$ were designed to locate all the closed loop poles in the same location of the negative real axis (i.e. $k_{p}=p^{2} / A$ and $\left.k_{d}=(2 p-B) / A\right)$ as the proposed controller. The values of the motor parameters used in the simulations are: $A=92.2339 \mathrm{~N} /(\mathrm{V} \mathrm{kg} \mathrm{m}) ; B=18.8192 \mathrm{~N} /(\mathrm{kg} \mathrm{m})$; $k=0.18 \mathrm{~N} \mathrm{~m} / \mathrm{V} ; n=156$ and $\Gamma_{c}=17.7840 \mathrm{~N} \mathrm{~m}$. The voltage compensation term caused by the Coulomb friction is therefore $\Gamma_{c} /\left(J n^{2} A\right)=0.57 \mathrm{~V}$. The servo amplifier accepts control inputs from the computer in the range of $[-10$, $10] \mathrm{V}$. The time sampling used in the simulations is $2 \times 10^{-3} \mathrm{~s}$. The gains of the controller can be designed by locating the poles in a reasonable location of the negative real axis. In our numerical simulations the closed loop poles have been located at $p=-95 \mathrm{rad} / \mathrm{s}$, and the value of the set of coefficients $\left\{k_{1}, k_{0}\right\}$ were obtained from the expressions found in (19). At the beginning of the simulation, the position and the velocity of the motor gear are defined by $\theta_{m}(0)=0$ and $\dot{\theta}_{m}(0)=0$, respectively, and the system is controlled to follow a desired trajectory prescribed by $\theta_{m}^{*}(t)$. Two different trajectories are used to
Table 2

Bezier trajectory - performances of control methods.

\begin{tabular}{llll}
\hline Control method & ISE & IAE & ITAE \\
\hline $\begin{array}{c}\text { PD + Coulomb } \\
\text { compensation }\end{array}$ & $0.51 \times 10^{-3}$ & $11.70 \times 10^{-3}$ & $7.19 \times 10^{-3}$ \\
$\begin{array}{c}\text { Robust feedforward } \\
\text { PD }\end{array}$ & $0.46 \times 10^{-3}$ & $6.22 \times 10^{-3}$ & $1.79 \times 10^{-3}$ \\
& & & \\
\hline
\end{tabular}

show the performance of the proposed controller, which are: (a) a sinusoidal trajectory given by $\theta_{m}^{*}(t)=M_{A} \sin$ $(\omega t)+M_{B}$ rad with $M_{A}=\pi / 12 \mathrm{rad}, M_{B}=\pi / 36 \mathrm{rad}$ and $\omega=1 \mathrm{rad} / \mathrm{s}$; and (b) It is supposed that the objective is to transfer the system from an initial equilibrium motor gear position to a final equilibrium motor gear position. A smooth nominal trajectory $\theta_{m}^{*}(t)$, is then prescribed during a finite interval of the form $\left[t_{i}, t_{f}\right]=[0,2] \mathrm{s}$, from an initial value $\theta_{m}^{*}\left(t_{i}\right)=\bar{\theta}_{m i}=\pi / 18$ rad to a desired final value $\theta_{m}^{*}\left(t_{f}\right)=\bar{\theta}_{m f}=\pi / 2 \mathrm{rad}$. We set, for instance 


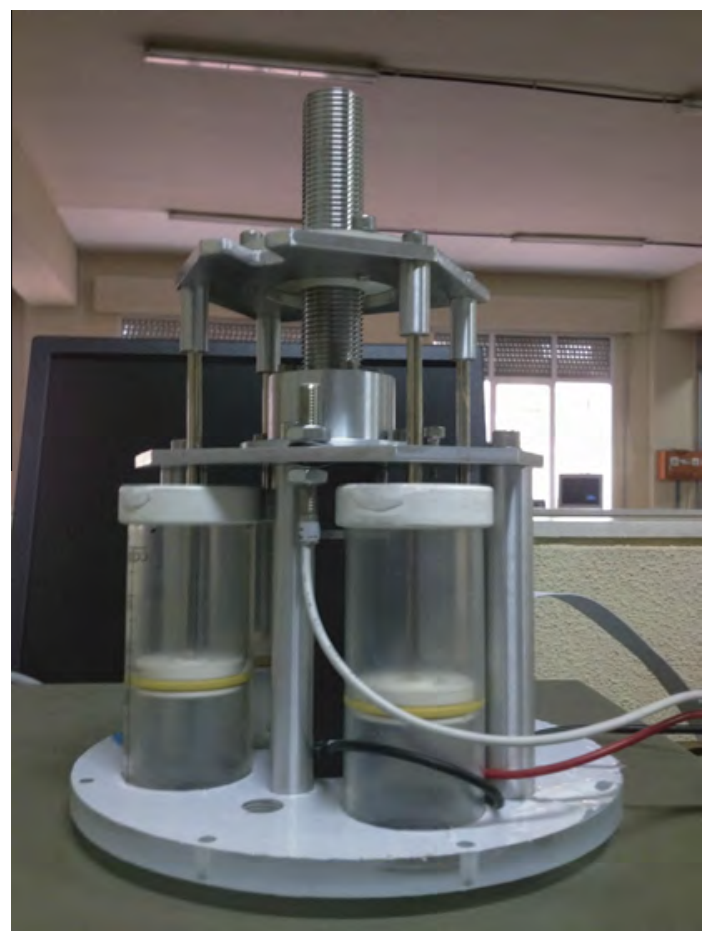

Fig. 13. Real DC motor platform.

$\theta_{m}^{*}(t)=\bar{\theta}_{m i}+\left(\bar{\theta}_{m f}-\bar{\theta}_{m i}\right) \varphi\left(\tau, t_{f}, t_{i}\right)$,

where $\varphi\left(\tau, t_{f}, t_{i}\right)$ is a Bezier polynomial that smoothly interpolates between 0 and 1 in the time interval $\left[t_{i}, t_{f}\right]$ and $\tau=\left(t-t_{i}\right) /\left(t_{f}-t_{i}\right)$. A 10-th order Bezier polynomial is chosed: $r_{1}=252 \quad r_{2}=1050 \quad r_{3}=1800$,

$r_{4}=1575 \quad r_{5}=700 \quad r_{6}=126$,

which guarantees that $\varphi\left(0, t_{i}, t_{f}\right)=0, \varphi\left(1, t_{i}, t_{f}\right)=1$ and $\left.\frac{d^{k} \varphi\left(\tau, t_{i}, t_{f}\right)}{d \tau^{k}}\right|_{\tau=0}=\left.\frac{d^{k} \varphi\left(\tau, t_{i}, t_{f}\right)}{d \tau^{k}}\right|_{\tau=1}=0$, for $k=1,2,3,4$.

Simulations are conducted in which an error of the order of $60 \%$ in the voltage compensation term caused by the Coulomb friction (i.e. $\Gamma_{c e} /\left(J n^{2} A\right)=0.228 \mathrm{~V}$ ) and errors of the order of $20 \%$ of the motor parameters $A$ and $B$ are assumed (i.e. $A_{c}=1.2 A$ and $B_{c}=1.2 B$ ). It should be noted that noises and errors which corrupt the measuring data are always found in real applications. In our particular application, the encoder in charge of the measurement of the magnitude $\theta_{m}$ and the amplifier which supplies the input voltage $u$ are not infinitely precise measurement systems. Noises are therefore taken into consideration in the control system owing to the limited precision apparatus. In particular it is supposed that the measurements of the magnitudes $\theta_{m}$ and $u$ are corrupted with an additive gaussian noise $N(0, \varsigma)$, denoted in Fig. 3 and Fig. 4 as $n_{\theta_{m}(t)}$ and $n_{u}(t)$ respectively, whose standard deviation is $\varsigma=10^{-3}$.

Furthermore, the synthesis of the proposed control requires the feeding back of the measured output $\theta_{m}$ and the estimation of its first and second order time derivatives, $\dot{\theta}_{m e}$ and $\ddot{\theta}_{\text {me }}$ according to (30). We propose generating the time derivatives of the measured output signal, $\dot{\theta}_{m e}(t)$ and $\ddot{\theta}_{m e}(t)$, with a truncated Taylor series expansion of $\theta_{m}(t)$, up to a fourth order around the re-initialization time $t_{r}$ expressed in terms of time varying linear filters in agreement with (11). Two parallel estimation processes are also launched with a specified alternating time duration, so that a better final estimation than that obtained with only one identifier subject to a re-initialization mechanism is

$\varphi\left(\tau, t_{i}, t_{f}\right)= \begin{cases}0 & \text { for } t<t_{i}, \\ \tau^{5}\left[r_{1}-r_{2} \tau+r_{3} \tau^{2}-r_{4} \tau^{3}+r_{5} \tau^{4}-r_{6} \tau^{5}\right] & \text { for } t_{i} \leqslant t \leqslant t_{f} \\ 1 & \text { for } t>t_{f},\end{cases}$

where

ensured. They are re-initialized every $T=0.4 \mathrm{~s}$ within a time separation between estimators of $0.2 \mathrm{~s}$. The first and

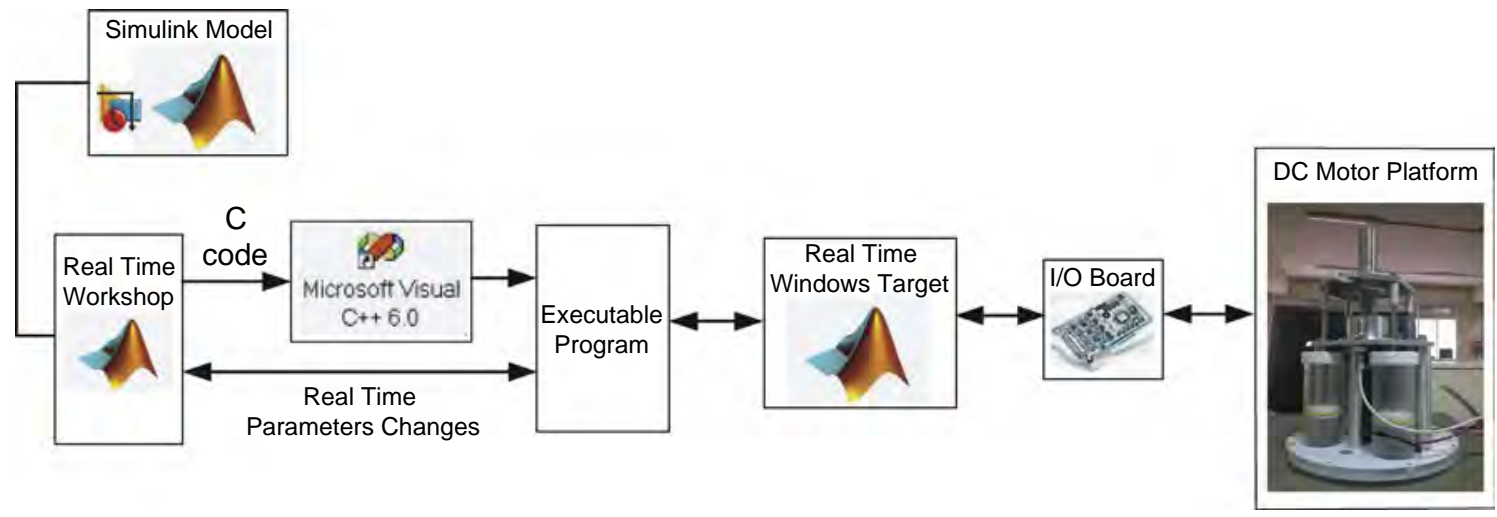

Fig. 14. Control system development flow diagram. 


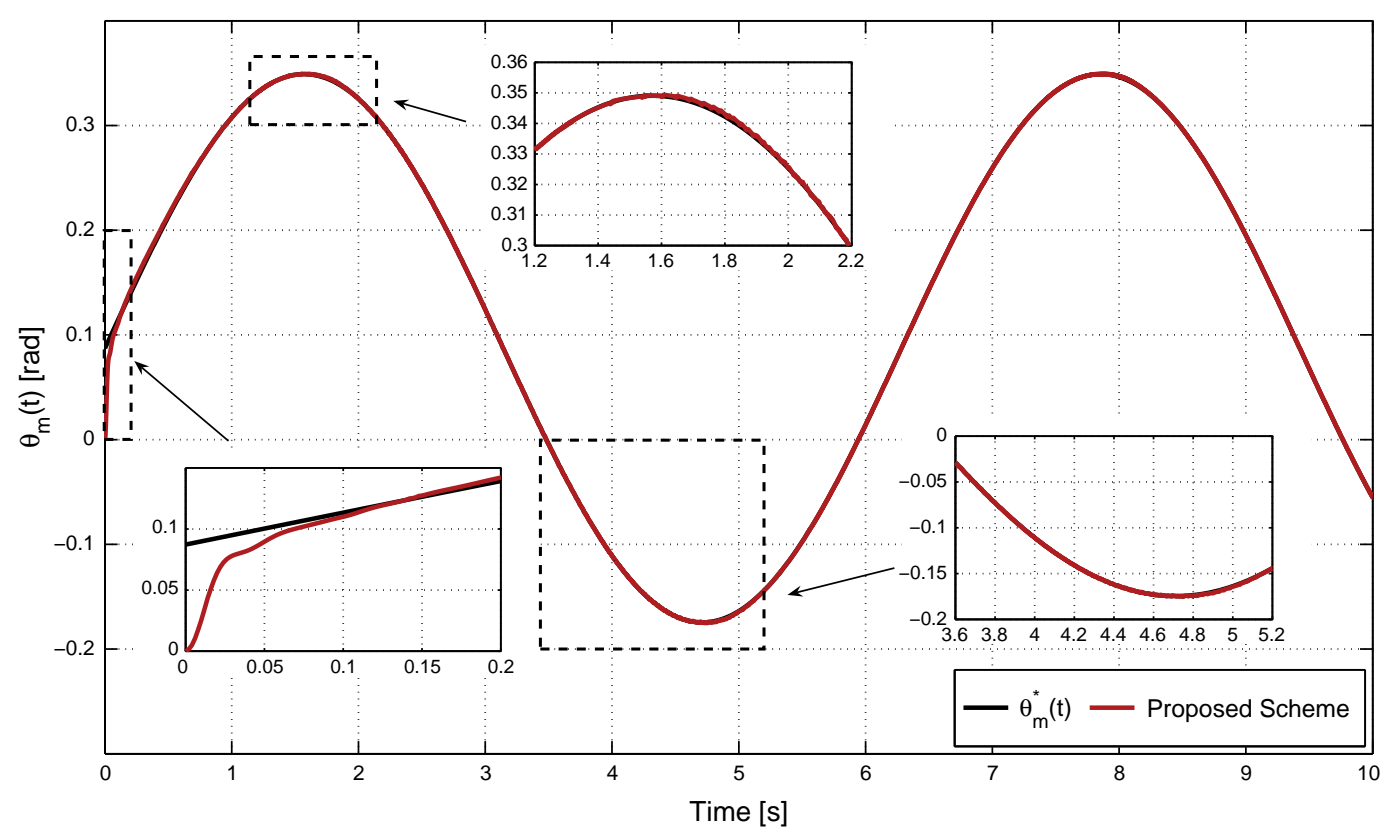

Fig. 15. Sinusoidal trajectory - experimental evolution of the DC motor position motor gear, $\theta_{m}$, using the robust feedforward PD controller when large initial errors appear and when the Coulomb's friction effects are not precisely known.

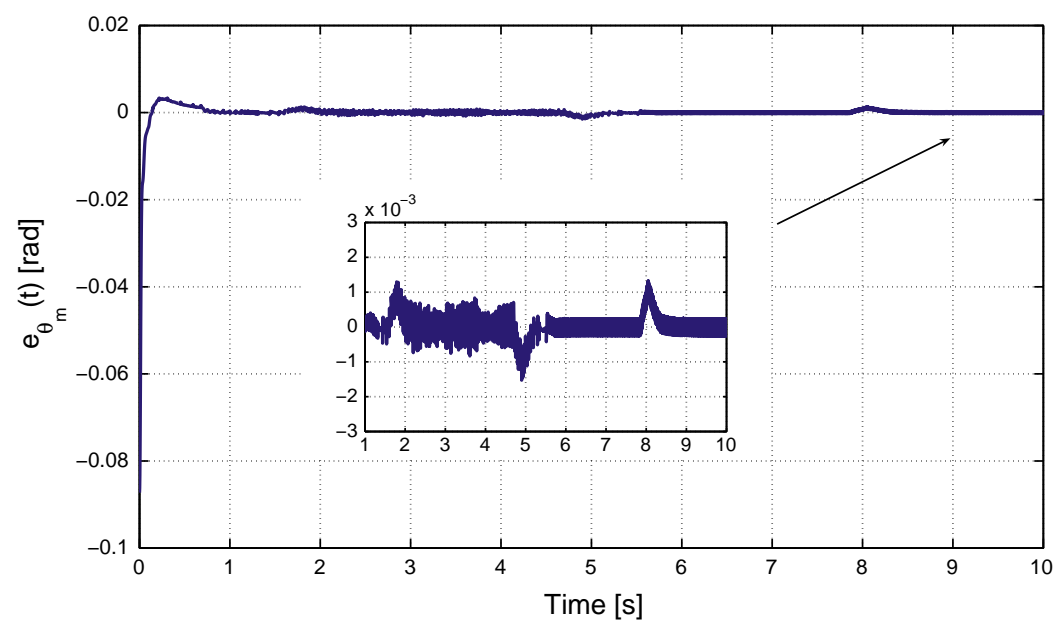

Fig. 16. Sinusoidal trajectory - experimental evolution of the tracking error, $e_{\theta_{m}}=\theta-\theta_{m}$, using a PD controller with Coulomb compensation and the robust feedforward PD controller when confronted with the appearance of large initial errors, imprecise knowledge of the Coulomb friction effects, noisy measurements and controller gain mismatches.

second derivatives in the first $\epsilon$-interval $(\epsilon=0.1 \mathrm{~s})$ are evaluated by computing dirty time derivatives via the transfer functions $P_{1}(s)=s /(0.005 s+1)$ and $P_{2}(s)=s^{2} /(0.005 s+1)^{2}$, respectively.

\subsection{Sinusoidal trajectory - robustness with regard to large} initial errors, inexact knowledge of the effects produced by the Coulomb friction, noisy measurements and controller gain mismatches

Fig. 5 depicts the numerical results of the closed loop controllers when the objective is to track a sinusoidal output reference trajectory in the presence of the sources of uncertainty previously described. At time $t=0.1 \mathrm{~s}$, the controller is updated with the estimated value of the voltage compensation caused by the Coulomb friction effects canceling the perturbation. Fig. 6 illustrates the trajectory tracking error, $e_{\theta_{m}}$, using the proposed controller and using the PD control with Coulomb compensation. As will be observed, despite all the desired effects considered, the feedback controller corrects the motion of the DC motor, guides the state errors to a small neighborhood of zero and compensates the errors in the model parameters.

Fig. 7 depicts the estimation of the voltage compensation term, $\hat{d}$ using the proposed controller and the input control, $u(t)$, using the proposed controller and using the 

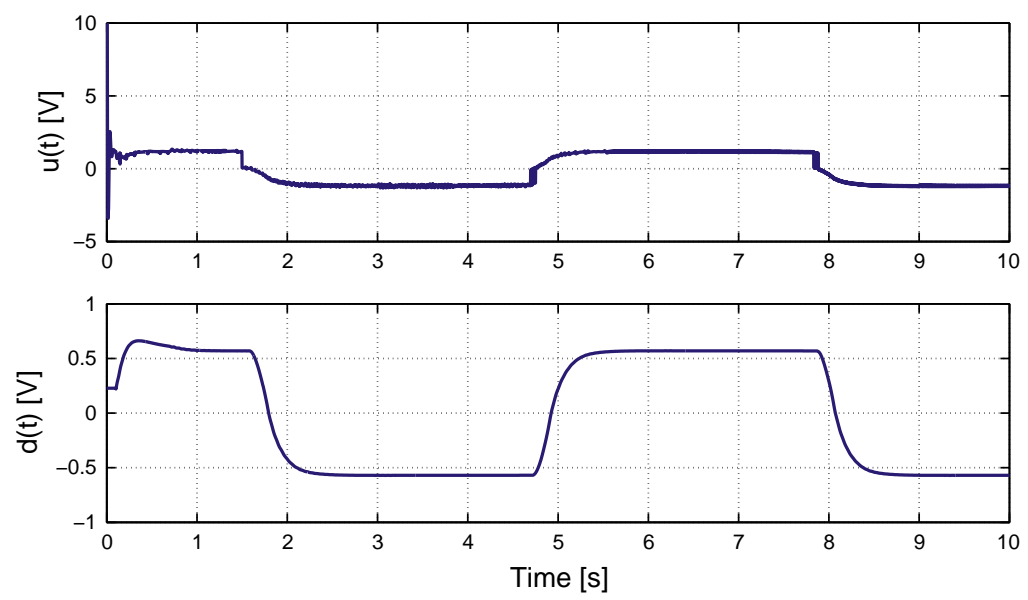

Fig. 17. Sinusoidal trajectory - experimental estimation of the parameter, $d(t)$, and input control, $u(t)$, using the robust feedforward PD controller when confronted with the appearance of large initial errors, imprecise knowledge of the Coulomb friction effects, noisy measurements and controller gain mismatches.
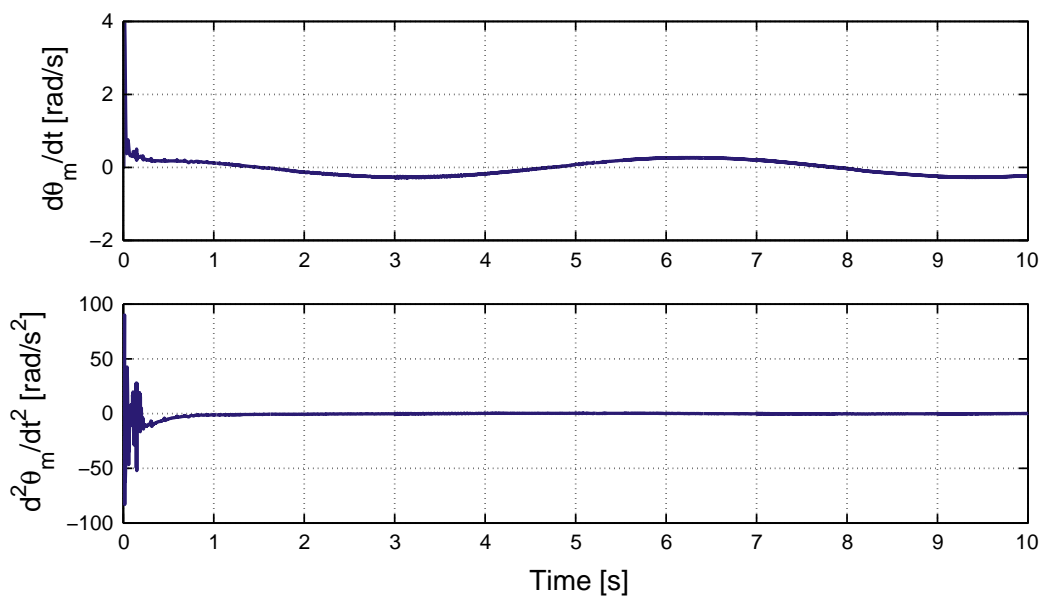

Fig. 18. Sinusoidal trajectory - experimental estimation of the DC motor gear velocity, $\dot{\theta}_{m}$ and estimation of the DC motor gear acceleration, $\ddot{\theta}_{m}$ when confronted with the appearance of large initial errors, imprecise knowledge of the Coulomb friction effects, noisy measurements and controller gain mismatches.

Table 3

Performances of the proposed control methods.

\begin{tabular}{llll}
\hline Trajectory & ISE & IAE & ITAE \\
\hline Sinusoidal & $0.99 \times 10^{-4}$ & $5.42 \times 10^{-3}$ & $1.16 \times 10^{-2}$ \\
Bezier & $0.41 \times 10^{-3}$ & $5.21 \times 10^{-3}$ & $1.33 \times 10^{-3}$ \\
\hline
\end{tabular}

PD control with Coulomb compensation. It will be noticed that the change in the sign of the velocity of the motor produces a change in the sign of the Coulomb friction term which is perfectly captured by the algebraic disturbance observer in the estimation of the parameter $\hat{d}$. What is more, the control input $u(t)$ is smaller and smoother with the proposed control law and the results achieved using the PD controller with Coulomb compensation illustrate an error signal in steady state which is not null.
Fig. 8 shows the evolution of the first and second time derivatives, $\dot{\theta}_{m e}$ and $\ddot{\theta}_{m e}$, using the algebraic derivative estimator and using a simple finite difference with a low pass filter of the velocity and acceleration of the motor gear. Upon comparing the performance of the overlapping algebraic derivative estimations with those obtained using finite difference with a low pass filter, it was concluded that the addition of the algebraic derivative estimations improves the behavior of the whole system in terms of low control effort and better stabilization and tracking trajectory tasks.

Additionally, the performances of the control methods have been measured in terms of the integral squared tracking error, $I S E=\int_{t_{A}}^{t_{B}} e_{\theta_{m}}^{2}(t) \mathrm{d} t$, the integral absolute tracking error, $I A E=\int_{t_{A}}^{t_{B}}\left|e_{\theta_{m}}(t)\right| \mathrm{d} t$, and the integral time absolute tracking error, ITAE $=\int_{t_{A}}^{t_{B}} t\left|e_{\theta_{m}}(t)\right| \mathrm{d} t$, where $t_{A}=0 \mathrm{~s}$ and $t_{B}=10 \mathrm{~s}$ denote the initial and final time of the simulation. The ISE and the IAE criterions will treat all the tracking 


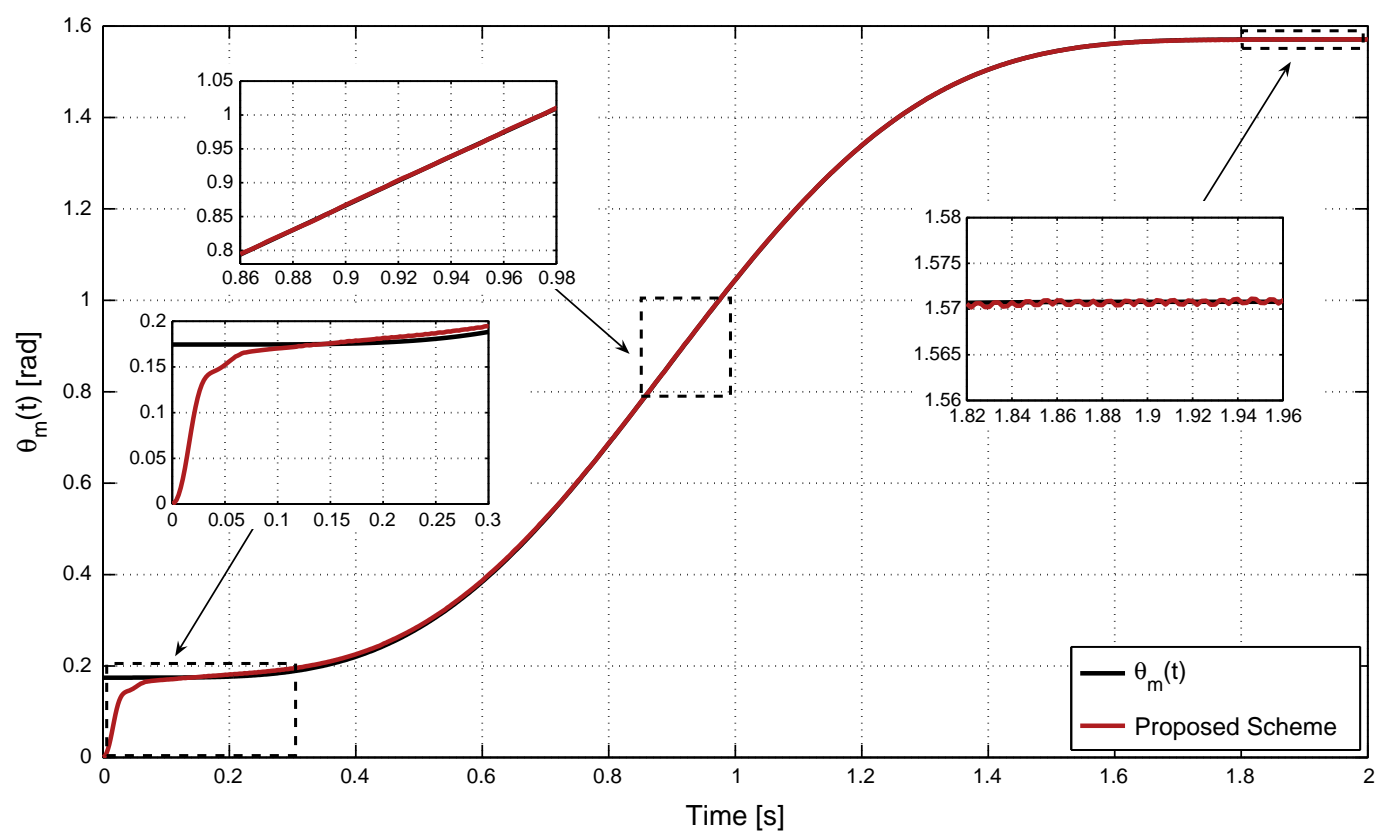

Fig. 19. Bezier trajectory - experimental evolution of the DC motor position motor gear, $\theta_{m}$, using the robust feedforward PD controller when large initial errors appear and when the Coulomb's friction effects are not precisely known.

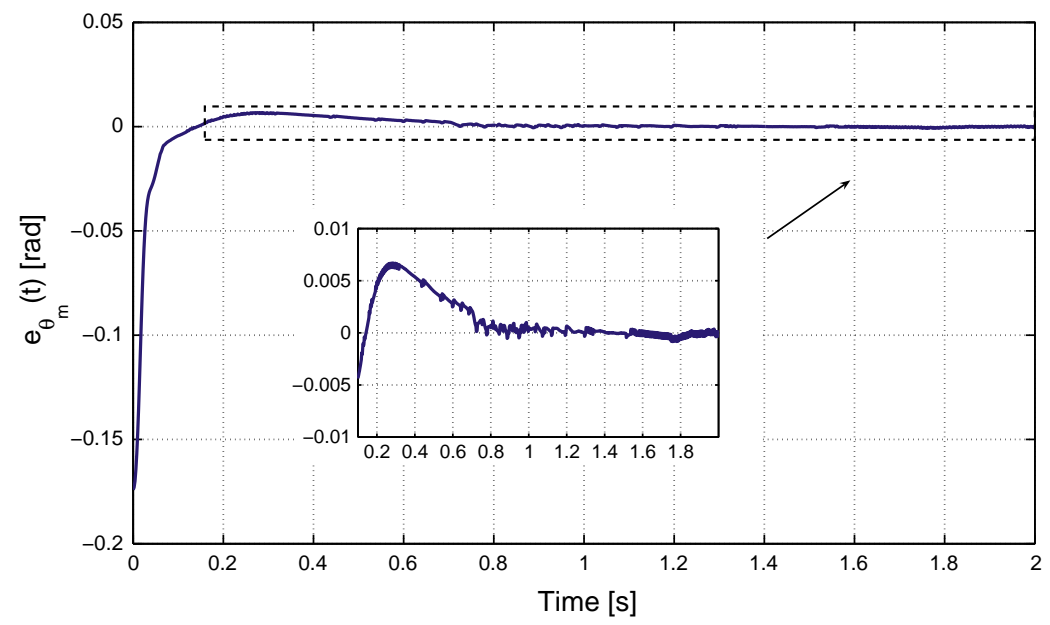

Fig. 20. Bezier trajectory - experimental evolution of the tracking error, $e_{\theta_{m}}=\theta-\theta_{m}$, using a PD controller with Coulomb compensation and the robust feedforward PD controller in the face of large initial errors appear, unprecise knowledge of the Coulomb's friction effects, noisy measurements and controller gain mismatches.

errors in a uniform manner. However, the ITAE criterion, as time appears as a factor, will heavily penalize errors that occur late in time but ignore errors that occur early in time. The results achieved are illustrated in Table 1, showing a better performance of the proposed control.

5.2. Rest-to-rest trajectory - robustness with regard to large initial errors, inexact knowledge of the effects produced by the Coulomb friction, noisy measurements and controller gain mismatches

Figs. 9-12 depict the numerical results of the closed loop controllers when the objective is to track a Bezier polynomial based interpolating, or rest to rest, trajectory in the presence of the sources of uncertainty previously described. At time $t=0.1 \mathrm{~s}$ the controller is updated with the estimated value of the voltage compensation caused by the Coulomb friction effects canceling the perturbation. As in the case of the sinusoidal trajectory, the use of the proposed robust control algorithm smooths and improves the behavior of the whole system for both the stabilization and the trajectory tracking tasks. The use of the algebraic derivative estimation in the control algorithm additionally allows the problems of control input saturations to be avoided in a substantial manner, thus allowing the system to obtain a better stabilization and tracking performances. 

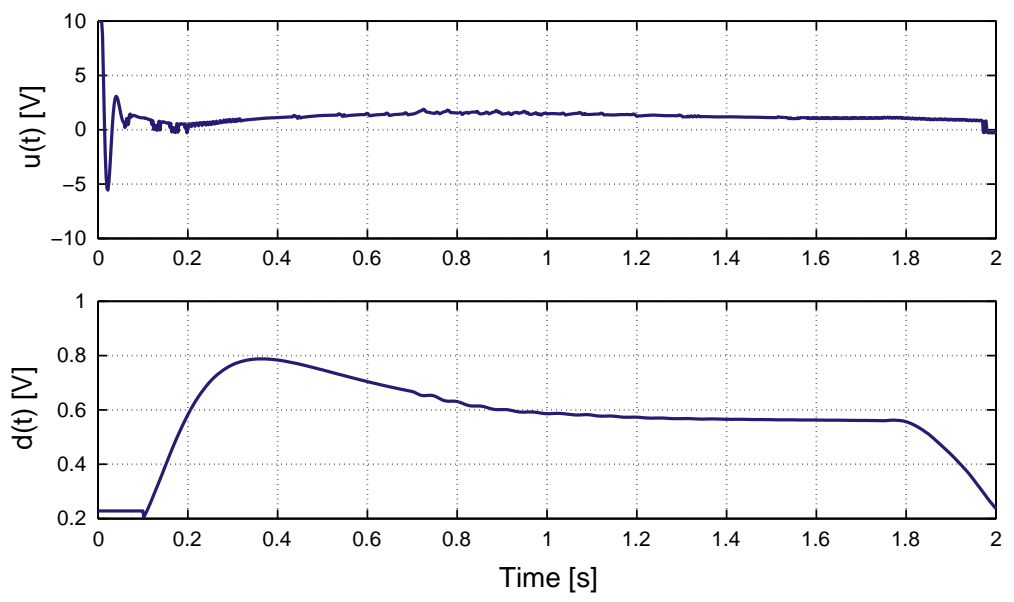

Fig. 21. Bezier trajectory - experimental estimation of the parameter, $d(t)$, and input control, $u(t)$, using the robust feedforward PD controller in the face of large initial errors appear, unprecise knowledge of the Coulomb's friction effects, noisy measurements and controller gain mismatches.
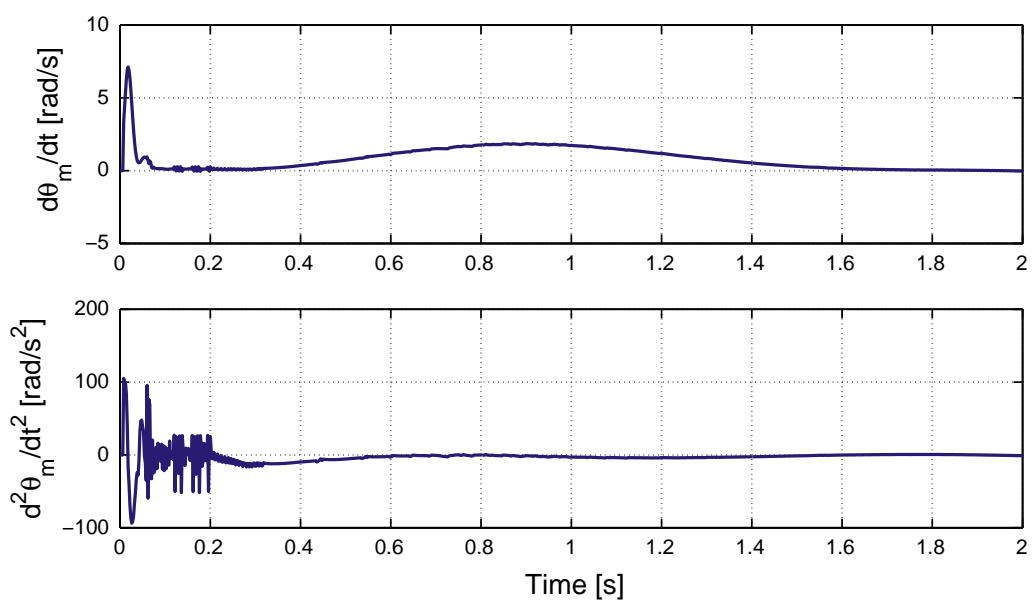

Fig. 22. Bezier trajectory - experimental estimation of the DC motor gear velocity, $\dot{\theta}_{m}$ and estimation of the DC motor gear acceleration, $\ddot{\theta}_{m}$ in the face of large initial errors appear, unprecise knowledge of the Coulomb's friction effects, noisy measurements and controller gain mismatches.

The results achieved in terms of ISE, IAE and ITAE with $t_{A}=0 \mathrm{~s}$ and $t_{B}=2 \mathrm{~s}$ are illustrated in Table 2 , showing a better performance of the proposed control.

\section{Experimental results}

This section provides a brief explanation of the experimental platform and the experimental design. The robust feedforward PD controller previously described is applied to a real DC motor. The experimental results obtained are described and validated.

\subsection{Experimental setup}

The DC motor used in the experiments is depicted in Fig. 13. In the prototype represented in this figure, the DC motor is mounted to control the buoyancy of a submerged platform by changing the volume of four small controlled pistons. The added changes of the pistons' volumes are carried out with longitudinal displacements.
The control of this platform must be very accurate because small changes in the positions of the pistons largely affect the buoyancy force. The control of this prototype is one of the future applications of our current research. The DC motor used is a DC motor Maxon RE40 ø40 mm, Graphite Brushes 150Watt which has a reduction ratio $n=156$ and an electromechanical constant $k=0.18 \mathrm{Nm} / \mathrm{V}$. The motor shaft is capable of turning either clockwise or counterclockwise around the $Z$ axis. A servo amplifier is used to supply the input voltage to the DC motor. This amplifier accepts control inputs from the computer in the range $[-10,10] \mathrm{V}$. The sensor system is integrated using an encoder, embedded in the motor, which makes it possible to know the motor position with a precision of $7 \times 10^{-5} \mathrm{rad}$. The sample interval in the signal processing support platform was set at $2 \times 10^{-3} \mathrm{~s}$. The other real values of the experimental set-up are: $A=92.2339 \mathrm{~N} /(\mathrm{V} \mathrm{kg} \mathrm{m})$; $B=18.8192 \mathrm{~N} /(\mathrm{kg} \mathrm{m})$ and $\Gamma_{C}=17.7840 \mathrm{~N} \mathrm{~m}$.

The control law implemented in the PC (operating in a Windows $^{\circledR}$ environment) uses software tools from 
Mathworks ${ }^{\circledR}$. These include MATLAB ${ }^{\circledR}$, Simulink, Control Toolbox, Real Time Workshop ${ }^{\circledR}$ (RTW), Real Time Windows Target $^{\circledR}$ (RTWT) and Visual $\mathrm{C}^{++}$Professional $^{\circledR}$. The steps necessary to obtain the executable file from the control law model are shown in Fig. 14. MATLAB ${ }^{\circledR}$ acts as the application host environment in which the other Mathworks ${ }^{\circledR}$ products run. This package offers extensive state-of-theart control design toolboxes. Simulink ${ }^{\circledR}$ provides a user friendly well structured graphical interface for the implementation of the control law. Real Time Workshop ${ }^{\circledR}$ automatically builds a $\mathrm{C}^{++}$source program from the Simulink Model. The $\mathrm{C}^{++}$Compiler ${ }^{\circledR}$ compiles and links the code created by Real Time Workshop ${ }^{\circledast}$ to produce an executable program. Real Time Windows Target ${ }^{\circledR}$ communicates with the executable program acting as the Control Program, and interfaces with the hardware device though the I/O Board.

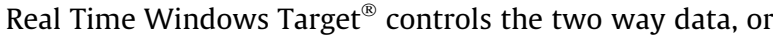
signal flow, to and from the model (which is now an executable program), and to and from the $\mathrm{I} / \mathrm{O}$ Board. The advantage of this approach is that the designer only needs to model the process, using the graphics tools available in Simulink $^{\circledR}$, without having to be concerned with the mechanics of communication to and from the control platform.

\subsection{Experiments}

The performance of the proposed controller was verified by carrying out experimental studies on the DC motor platform. Figs. 15 and 19 illustrate the experimental evolution of the trajectory tracking of the motor gear when the objective is to track a sinusoidal trajectory and a rest-torest trajectory. Figs. 16 and 20 show the trajectory tracking errors, $e_{\theta_{m}}$, of the previously described trajectories. It can be verified that the proposed approach exhibits an excellent performance and after $t=0.1 \mathrm{~s}$, which produces the update process of the estimation parameter $\hat{d}(t)$, the tracking error is substantially reduced to a small neighborhood of zero which is in accordance with the above numerical simulations. Figs. 17 and 21 depict the control input needed to track the reference trajectories selected and the estimation of the voltage compensation term, $\hat{d}$. It will be observed that after $t=0.1 \mathrm{~s}$, the control input depicts a small and smooth behavior. What is more, both signals, the control input and the parameter estimation are in accordance with the above numerical simulations. Finally, Figs. 18 and 22 illustrate the evolution of the first and second time derivatives, $\dot{\theta}_{m e}$ and $\ddot{\theta}_{m e}$, showing a high correspondence with the above numerical simulations. The results achieved in terms of ISE, IAE and ITAE are depicted in Table 3, showing a better performance of the proposed control which is in accordance with the numerical simulations.(mfloat Figs. 19-22)

\section{Conclusions and future work}

In this work, a robust feedforward PD controller design approach has been proposed for the efficient stabilization and reference trajectory tracking of DC motors. The proposed methodology requires only the measurement of the angular position of the motor gear and the voltage input to the motor. It is based on the use of an overlapping implementation of the algebraic derivative estimation method to estimate the time derivatives of the angular position of the motor gear and the inclusion of a disturbance estimator based on the algebraic derivative estimation method. The estimated values achieved from the two shifted estimators by using the convergent estimator is used while the other estimator is starting to diverge and is thus being properly reset. The outcome of this technique is a considerable acceleration of the convergence of the computation transients that occur just after the estimation resettings. A second enhancement is the invariant filtering effects for the overall attenuation of zero mean high frequency noise effects. The performance of the proposed controller has been simulated and tested on an actual laboratory prototype. The experiments had a high performance with regard to previous controllers in the stabilization and trajectory tracking tasks. Finally, this methodology is well suited to confronting important problems of control systems. Its versatility and ease of implementation make the controller suitable to be robustly applied in a wide range of real engineering applications such as the buoyancy control of a submerged platform [34], the control of stair-climbing mobility systems [35-41] or the control of electronic converters [42]. This will be the topic of our future publications.

\section{Acknowledgment}

This work has been partially supported by the Spanish Ministerio de Ciencia e Innovación under Grant DPI201124113.

[1] S.E. Lyshevski, Nonlinear control of mechatronic systems with permanent-magnet DC motors, Mechatronics 9 (1999) 539-552.

[2] S.E. Lyshevski, Control of Electrical Drives, Springer, 2001.

[3] J.O. Jang, G.J. Jeon, A parallel neuro-controller for DC motors containing nonlinear friction, Neurocomputing 30 (2000) 233-248.

[4] C.P. Tang, P.T. Miller, V.N. Krovy, J.-C. Ryu, S.K. Agrawal, Differentia flatness-based planning and control of a wheeled mobile manipulator theory and experiment, IEEE/ASME Transactions on Mechatronics 16 (4) (2011) 768-773.

[5] F. Wang, X. Zhao, D. Zhang, Z. Ma, X. Jing, Robust and precision control for a directly-driven XY table, Proceedings of the Institution of Mechanical Engineers, Part C: Journal of Mechanical Engineering Science 225 (5) (2011) 1107-1120.

[6] V.C. Mummadi, Steady-state and dynamic performance analysis of PV supplied DC motors fed from intermediate power converter, Solar Energy Mater Solar Cells 61 (2000) 365-381.

[7] M. Mahfouf, C.H. Kee, M.F. Abbod, D.A. Linkens, Fuzzy logic based anti-sway control design for overhead cranes, Neural Computing \& Applications 9 (2000) 38-43.

[8] Y. Yavin, Control of the motion of a disk rolling on a plane curve, Computers \& Mathematics with Applications 54 (11-12) (2007) 1329-1340.

[9] H. Seong Lee, M. Tomizuka, Robust motion controller design for high-accuracy positioning systems, IEEE Transactions on Industrial Electronics 43 (1996) 48-55.

[10] I. Elker, Open loop and closed loop experimental on line identification of a three mass electromechanical system, Mechatronics 14 (2004) 549-565.

[11] H. Olsson, K. Amström, C.C. de Wit, Friction models and friction compensation, European Journal of Control 4 (1998) 176-195. 
[12] K. Nouri, R. Dhaouadi, N.B. Braiek, Adaptive control of a nonlinear dc motor drive using recurrent neural networks, Applied Soft Computing 8 (2008) 371-382.

[13] I. Elker, Sliding mode control with PID sliding surface and experimental application to an electromechanical plant, ISA Transactions 45 (1) (2006) 109-118.

[14] M. Fliess, H. Sira-Ramírez, An algebraic framework for linear identification, ESAIM, Control, Optimization and Calculus of Variations 9 (2003) 151-168.

[15] M. Fliess, H. Sira-Ramírez, Closed-loop identification parametric identification for continuous time linear systems, in: H. Granier, L. Wang (Eds.), Continuous Time Model Identification from Sampled Data, Springer, Berlin, 2007.

[16] G. Mamani, J. Becedas, H. Sira-Ramírez, V. Feliu, Open-loop algebraic identification method for DC motor, in: European Control Conference, Greece, 2007.

[17] H. Sira-Ramírez, E. Barrios Cruz, R. Márquez Contreras, Fast adaptive trajectory tracking control for a completely uncertain DC motor via output feedback, Computación y Sistemas 12 (4) (2009) 397-408.

[18] J. Reger, H. Sira-Ramírez, M. Fliess, On-non-asymptotic state estimation of nonlinear systems, in: Proc. 44th IEEE Conference on Decision and Control (CDC'05), Seville, 2005.

[19] J. Reger, P. Mai, H. Sira-Ramírez, Robust algebraic state estimation of chaotic systems, in: IEEE 2006 CCA/CACSD/ISIC, Munich, Germany, 2006.

[20] S. Diop, M. Fliess, Nonlinear observavility, identifiability and persistent trajectories, in: Proceedings of the 36th IEEE Conference on Decision and Control, Brighton, England, 1991, pp. 714-719.

[21] S. Diop, J.W. Grizzle, F. Chaplais, On numerical differentiation algorithms for nonlinear estimation, in: Proceedings of the 39th IEEE Conference on Decision and Control, Sydney, Australia, 2000, pp. $1133-1138$.

[22] S. Diop, J.W. Grizzle, P.E. Moraal, A. Stefanopoulou, Interpolation and numerical differentiation for observer design, in: Proceedings of the American Control Conference, Baltimore, USA, 1994, pp. 1329-1333.

[23] A. Levant, Robust exact differentiation via sliding mode technique, Automatica 34 (3) (1998) 379-384.

[24] M. Fliess, C. Join, H. Sira-Ramírez, Non-linear estimation is easy, International Journal of Modelling, Identification and Control 4 (1) (2008) 12-27.

[25] M. Fliess, Analyse non standard du bruit, C.R. Acad. Sci. Paris, 2006, p. Ser. I 342.

[26] J. Zehetner, J. Reger, M. Horn, A derivative estimation toolbox based on algebraic methods - theory and practice, 16th IEEE International Conference on Control Applications, Part of IEEE Multi-Conference on Systems and Control (2007) 331-336.

[27] M. Horn, J. Zehetner, A brake-testbench for research and education, 16th IEEE International Conference on Control Applications, Part of IEEE Multi-Conference on Systems and Control (2007) 444-448.
[28] A. Gensior, J. Weber, J. Rudolph, H. Güldner, Algebraic parameter identification and asymptotic estimation of the load of a boost converter, IEEE Transactions on Industrial Electronics 55 (9) (2008) $3352-3360$.

[29] V. Feliu, K. Rattan, H.B. Brown, Control of flexible arms with friction in the joints, IEEE Transactions on Robotics and Automation 9 (4) (1993) 467-475.

[30] V. Feliu, J.A. Somolinos, C. Cerrada, J.A. Cerrada, A new control scheme of single-link flexible manipulators robust to payload changes, Journal of Intelligent and Robotic Systems 20 (2-4) (1997) 349-373.

[31] J.A. Somolinos, V. Feliu, L. Sánchez, J.A. Cerrada, Modeling and control of a new three degree-of-freedom flexible arm with simplified dynamics, Proceedings of the 1999 IEEE International Conference on Robotics and Automation 1 (1999) 435-440.

[32] V. Feliu, F. Ramos, Strain gauge based control of single-link flexible very lightweight robots robust to payload changes, Mechatronics 15 (2005) 547-571.

[33] E. Pereira, J.R. Trapero, I.M. Díaz, V. Feliu, Adaptive input shaping for single-link flexible manipulators using an algebraic identification, Control Engineering Practice 20 (2) (2012) 138-147.

[34] J.A. Somolinos, A. López, L.R. Nuñez, R. Morales, Control of a novel underwater ocean current turbine, International Journal of Systems Control 3 (1) (2012) 13-18.

[35] R. Morales, V. Feliu, A. González, P. Pintado, Kinematic model of a new staircase climbing wheelchair and its experimental validation, The International Journal on Robotics Research 25 (9) (2006) 825841.

[36] A. González, R. Morales, V. Feliu, P. Pintado, Improving the mechanical design of new staircase wheelchair, Industrial Robot: An International Journal 34 (2) (2007) 110-115.

[37] R. Morales, A. González, V. Feliu, P. Pintado, Environment adaptation of a new staircase climbing wheelchair, Autonomous Robots 23 (2007) 275-292..

[38] R. Morales, A. González, V. Feliu, Optimized obstacle avoidance trajectory generation for a reconfigurable staircase climbing wheelchair, Robotics and Autonomous Systems 58 (2010) 97-114.

[39] R. Morales, J.A. Somolinos, J.A. Cerrada, Dynamic model of a stairclimbing mobility system and its experimental validation, Multibody Systems Dynamics 28 (2012) 349-367.

[40] R. Morales, J.A. Somolinos, J.A. Cerrada, Dynamic control for a reconfigurable stair-climbing mobility system, Robotica 31 (2) (2013) 295-310.

[41] R. Morales, J. Chocoteco, V. Feliu, H. Sira-Ramírez, Obstacle surpassing and posture control of a stair-climbing robotic mechanism, Control Engineering Practice 21 (2013) 604-621.

[42] R. Morales, J. Somolinos, C. Morón, A. García, Space-state robust control of a buck converter with amorphous core coil and variable load, Measurement 46 (10) (2013) 3863-3870. 